\title{
Review: Model particles in atmospheric optics
}

\author{
Michael Kahnerta, ${ }^{\mathrm{a} b}$, Timo Nousiainen ${ }^{\mathrm{c}}$, Hannakaisa Lindqvist ${ }^{\mathrm{d}}$ \\ ${ }^{a}$ Research Department, Swedish Meteorological and Hydrological Institute, Folkborgsvägen \\ 1, SE-601 76 Norrköping, Sweden \\ ${ }^{b}$ Department of Earth and Space Science, Chalmers University of Technology, SE-412 96 \\ Gothenburg, Sweden \\ ${ }^{c}$ Finnish Meteorological Institute, Earth Observation, P.O. Box 503, FI-00101 Helsinki, \\ Finland \\ ${ }^{d}$ Department of Physics, University of Helsinki, P.O. Box 48, FI-00014 Helsinki, Finland
}

\begin{abstract}
This review paper provides an overview over model geometries for computing light scattering by small particles. The emphasis is on atmospheric optics, although much of this review will also be relevant to neighbouring fields, in particular to astronomy. Various morphological particle properties are discussed, such as overall nonsphericity, pristine shapes, aggregation, and different forms of inhomogeneity, e.g. porous and compact inhomogeneous morphologies, as well as encapsulated aggregates. Models employed to reproduce the optical properties of complex particles range from strongly simplified to highly realistic and morphologically sophisticated model geometries. Besides reviewing the most recent literature, we discuss the idea behind models of varying degree of complexity with regard to the intended use of the models. Applications range from fundamental studies of light scattering processes to routine applications of particle optics look-up tables in operational modelling systems.
\end{abstract}

Keywords: Scattering, aerosols, ice clouds, mineral dust, black carbon, cosmic dust, regolith

\section{Introduction}

Light-matter interaction is among the most important processes in physics; it is exploited in numerous experimental techniques and extensively studied

Email address: michael.kahnert@smhi.se (Michael Kahnert) 
in different branches of theoretical physics. An important sub-set of such processes is the interaction of electromagnetic radiation with small particles. Light-scattering processes have numerous applications in atmospheric and planetary sciences, in the studies of solar-system objects [1], proto-planetary disks, exoplanets, the circumstellar environment of red giant stars, as well as the physics and chemistry of the interstellar medium (e.g. [2]). There are also important applications in ocean optics, biomedical optics [3], nano-optics [4], material science [5], process engineering, and combustion diagnostics [6].

In atmospheric physics one frequently encounters ensembles of aerosol particles or hydrometeors that exhibit a large range of different sizes, can have complex, non-spherical shapes, and possess varying, often heterogeneous chemical composition. A theoretical description of the interaction of light with such particle ensembles is a formidable problem. More often than not it can only be solved by introducing simplifying assumptions about the particle shapes.

The use of different model geometries in light scattering computations can sometimes give rise to scientific controversies. To take a concrete example, an ellipsoid model for describing Martian dust particles (e.g. [7]) may be questioned because of its simplicity; ellipsoids have little morphological resemblance to real Martian aerosols. Thus one may question the physical insights that can be gained from such a model. On the other hand, one could equally well criticise that this model is too complex, as a simpler spheroid model may, perhaps, be sufficiently accurate to reproduce the optical properties of Martian dust. The point of view we take here is that there is little meaning in discussing such questions decoupled from the question of the intended application of the model. Inversion methods for global remote sensing data sets (e.g. [8, 9]) or radiative transfer applications in climate models (e.g. [10]) often necessitate a pragmatic approach based on the use of drastically simplified model geometries. On the other hand, in more fundamental studies aimed at attaining a basic understanding of the relation between morphological and radiative particle properties, one often needs to afford the use of much more elaborate model geometries.

Besides providing an overview of the existing literature, one purpose of this review is to discuss the rationale behind different approaches and approximations to represent complex morphologies in particle optics models, and to discuss their range and limits of applicability with regard to the intended uses of the models. The discussion focuses on atmospheric particles. However, much of this review will also be relevant to other fields, especially 
to applications of light scattering in astronomy.

Section 2 will first discuss the basic rationale of using particle models of various degree of complexity in light scattering computations. Section 3 will provide a review of modelling approaches for different morphological features. In principle, we could have equally well divided that section into sub-sections for different types of particles, e.g. ice particles and aerosols of different chemical composition (secondary inorganics, black carbon, organics, salt, mineral dust). Alternatively, we could have based the review on the sources of the particles (anthropogenic, marine, wind-blown dust, biogenic, etc). Instead, we organised the discussion according to different morphological characteristics of particles, such as large-scale nonsphericity, small-scale surface roughness, irregularity, aggregation, inhomogeneity, etc. This is the most logical structuring, as we are mainly interested here in the connection of particle morphology and particle optics, and in how to represent morphologically complex features in optics models.

The main target audience of this review are practitioners. Those who want to obtain more detailed information on electromagnetic scattering theory may start by consulting $[11,12,13]$. Reviews of numerical light scattering methods can be found in $[14,15,16]$. Available light scattering computer codes are discussed in [17].

\section{Basic rationale of particle models in electromagnetic scattering}

The real world is a messy place. By their very nature, models attempt to focus on the essential aspects of a complex system, while omitting or simplifying less important processes and characteristics of the system under investigation. Atmospheric particles cover a large range of sizes, morphologies, and chemical compositions. Moreover, the position as well as the physical and chemical properties of each particle can change with time. It is usually neither possible nor desirable to compute optical properties by working with exact replicas of each and every particle encountered in a real particle ensemble. Thus, we are faced with the question how to represent complex particle morphologies in aerosol optics models. The choice of suitable model geometries is constrained by our incomplete knowledge of real-world particles, the computational resources we can afford, and the range of applicability of available numerical methods. Most importantly, the choice of model geometry will be determined by its intended application. 


\subsection{Applications of particle models to environmental modelling and remote sensing}

One class of applications comprises large modelling systems designed for routine use (e.g. operational services), such as climate models or inversion schemes for remote sensing data sets. In such applications one rarely employs morphologically detailed model particles. There are several reasons why such large modelling systems almost exclusively employ highly simplified model geometries for aerosol optics modelling. One major limitation is the computational burden involved in creating aerosol optics look-up tables for climate models or remote sensing retrieval methods. The large range of sizes and the high variability in the chemical composition of aerosols requires lookup tables that cover a large parameter space. This severely limits the level of detail one can afford in the light-scattering computations.

However, there are also other, equally important reasons that justify the use of highly simplified model particles. To understand these reasons, let us take, as an example, modelling approaches for the direct radiative effect of aerosols in climate models. The number of physical and chemical aerosol properties that are accounted for in climate models is highly limited. For instance, the most detailed modelling systems currently available (known as Earth-system models) contain a chemical transport model (CTM) with an aerosol dynamics module (e.g. [18]) coupled to an atmosphere-ocean circulation model. The CTM delivers the spatial distribution and temporal evolution of atmospheric aerosols, which are characterised by their mass concentration and number distribution and their size-resolved chemical composition. Based on this information, one can obtain the size-dependent refractive index of the aerosols in each grid cell of the 3-dimensional model domain, from which one can compute the aerosol optical properties. This information is fed into the climate model's radiative transfer scheme to compute the radiative forcing effect of aerosols on the atmosphere-ocean system. The salient point is that there are no processes in the model that deliver any information on particle shape (apart from the mixing state of different chemical components). The chemical composition is influenced by the particles' emission sources, chemical transformation processes, and by aerosol dynamic mixing processes, which are accounted for by the CTM. The size distribution results (for secondary particles) from dynamic processes, such as nucleation, condensation, and coagulation (which are described in the aerosol dynamics model), or (for sea salt and wind-blown dust) from emission processes. It is also influenced by dry and wet deposition processes, all of which are accounted for 
in the model. However, the particle shapes of, e.g., desert dust particles are the result of aeolian erosion and stochastic collision processes that are not described in the model. In this situation, the logical approach for aerosol optics modelling is to employ a model that makes use of the aerosol information provided by the model (i.e., the size distribution and chemical composition), and to invoke suitable assumptions about the particle geometries.

By "suitable" we mean that such assumptions should be as simple as possible and as complex as necessary. The only requirement is that the model needs to reproduce the desired optical properties of real aerosol ensembles with sufficient accuracy. For climate models, this means that the model geometries need to yield correct estimates of the ensemble-averaged extinction cross section, single scattering albedo, and asymmetry parameter, i.e., those optical properties that are required as input to the radiation transport model. There is little to be gained by employing morphologically faithful, geometrically complex, and computationally highly demanding model geometries in such applications, unless one cannot find a simpler model geometry that can mimic the aerosol scattering and absorption properties of real aerosols with sufficiently high accuracy.

In remote sensing one often observes directional radiative characteristics at several wavelengths, which are strongly influenced by the differential scattering properties of particles and their spectral dependence. Remote sensing retrieval methods are algorithms that attempt to solve an ill-posed inverse problem. From satellite, air-borne, or ground-based observations of, e.g., directional spectral radiances or polarimetric properties, one tries to retrieve physical and chemical properties of aerosols or hydrometeors that reflect, attenuate, or emit radiation in the atmosphere. Observations of optical parameters usually do not contain sufficient information to fully retrieve the aerosols' physical properties and chemical composition (e.g. [19]). From a practical point of view, this makes it necessary to focus on retrieving those aerosol properties that have the strongest impact on the observed radiative and polarimetric properties. From a more scientific point of view, one wishes to retrieve aerosol properties that are linked to those dynamic and chemical processes that are most important for understanding the sources, evolution, and fate of aerosols in the atmosphere. The chemical composition and the size of the aerosols fit both of these criteria. On the other hand, the information content of remote sensing observations does not allow us to fully retrieve the particle morphology, nor would such information be very valuable at our current level of process understanding. Although particle shape can, con- 
ceivably, have an impact on processes such as dry deposition or the ability of aerosols to act as ice crystal nuclei, such details are not accounted for in existing CTMs. It is, therefore, only a slight exaggeration to say that particle shape is a nuisance rather than a source of useful extra information in remote sensing, given our present capabilities. This often justifies to account for particle non-sphericity by use of highly simplified model geometries. A somewhat less radical way to express this notion would be to advocate the design of inversion schemes that make flexible use of different particle models depending on the intended use of the retrieval products.

Needless to say, there are other applications of light scattering, such as in bio-optics, where retrieval of particle morphology can be extremely important. So the argument above for the use of simple model particles cannot be generalised. At the risk of repeating ourselves, we emphasise again that the rationale of a particular choice of model particle can only be discussed in the context of its intended application.

\subsection{Applications of particle models in fundamental electromagnetic scatter- ing studies}

Numerous studies aim at deepening our understanding of light scattering processes. The motivation for such studies may be

- to validate the usefulness of simpler model particles by comparing their optical properties to those of more complex, presumably more realistic model particles;

- to produce computational benchmarks or to test numerical methods;

- to selectively investigate the impact of specific morphological features on the optical properties of particles; or

- to study the morphology-dependent relation between the internal field inside the particle and the scattered field it generates (e.g. [20, 21, 22, 23]).

Just because these studies are dealing with more fundamental questions than more applied studies does not mean that the particle models automatically have to be more complex. The earliest studies of the effect of nonsphericity on optical properties focused on axisymmetric shapes such as spheroids (e.g. $[24,25,26,27,28,29,30,31]$, finite circular cylinders [32, 33], and 2DChebyshev particles $[34,35,36,37,38,39]$. Comparisons between the light 
scattering properties of such simple nonspherical geometries and those of homogeneous spheres were invaluable for gaining the first insights into the significance of nonsphericity for particle optics.

With our improving computational capabilities, the particle models have become more and more complex. A review of recent developments will be given in the next section. But before plunging into the details, let us continue our discussion of the rationale for the use of model particles of different degrees of complexity in fundamental light scattering studies. One may think that the best computational model would be based on the use of exact replicas of real-world particles. On the other hand, one may also be reluctant to call this a "model". The very concept of a model implies the idea of suitable approximations to a complex, realistic system. No one has expressed the rationale for approximations in physics more clearly than Arthur Eddington [40]:

"I conceive that the chief aim of the physicist in discussing a theoretical problem is to obtain 'insight' — to see which of the numerous factors are particularly concerned in any effect and how they work together to give it. For this purpose a legitimate approximation is not just an unavoidable evil; it is a discernment that certain factors - certain complications of the problem - do not contribute appreciably to the result. We satisfy ourselves that they may be left aside; and the mechanism stands out more clearly, freed from these irrelevancies. This discernment is only a continuation of a task begun by the physicist before the mathematical premises of the problem could be stated; for in any natural problem the actual conditions are of extreme complexity and the first step is to select those which have an essential influence on the result - in short, to hold the right end of the stick. The correct use of this insight, whether before or after the mathematical problem has been formulated, is a faculty to be cultivated, not a vicious propensity to be hidden from the public eye."

For our problem at hand, this means that a model particle is not necessarily better than others if it is more complex or more similar to real particles. More complex models introduce a larger number of parameters that need to be studied, or, in inverse problems, retrieved. Again, the main question is what the intended application is, what scientific question we are asking. 
The use of more or less exact replicas of realistic particles in light scattering computations can be seen as a numerical laboratory, rather than as a model. Such a theoretical laboratory does have distinct advantages over actual measurements, not least because one has complete control over all physical parameters of the particle. Such light scattering computations can be invaluable for the evaluation of simpler model particles (e.g. [41]). On the other hand, to gain physical insight into the relation between particle shape and optical properties, the use of more stylized geometries that are designed to selectively mimic specific morphological aspects can often be more rewarding. We shall repeatedly return to these questions in the following sections.

\subsection{Approximate theories}

Besides approximations of particle properties, simplifications of the scattering problem can also be achieved by employing computational methods based on approximate theory. Such methods can either be derived by introducing approximations in Maxwell's equations (e.g. $[14,15]$ ), or they can be obtained from ad hoc assumptions. In the former case, the limits of the validity of the methods are well-understood. As mentioned above, approximations can often provide valuable insights into physical processes. A discussion of the latter case and its drawbacks is given in [42]. Here we just wish to emphasize that models based on ad hoc assumptions typically have a highly limited capacity to illuminate the workings of the laws of physics in electromagnetic scattering processes. Their chief goal is to provide numerical methods that, under certain circumstances and for limited purposes, are able to fit the optical properties of complex particles. For the most part, we shall limit the discussion in this article to approximations of the particle geometry and dielectric properties.

\section{Modelling approaches for different particle morphologies}

\subsection{Small-scale surface roughness}

We start our review with small-scale surface roughness, because the discussion of this rather specific morphological feature will illustrate many of the points we addressed in the previous section. Solid-phase particles of non-pristine shapes frequently have rough surfaces. Mineral dust in planetary atmospheres or ice cloud particles onto which supercooled droplets have 

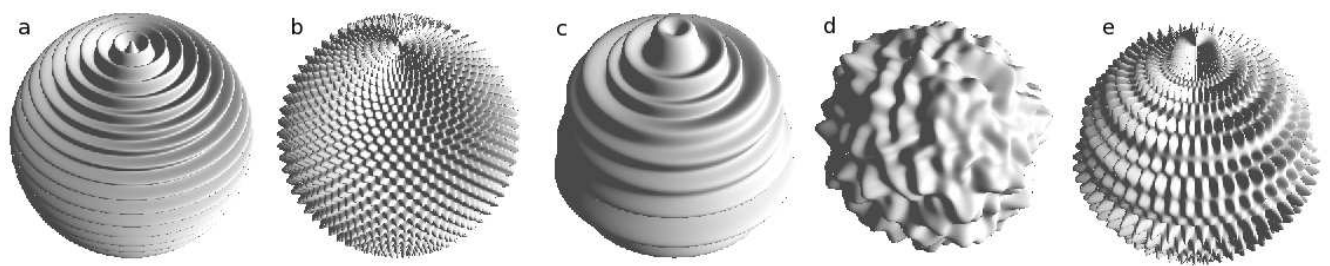

Figure 1: Models for small-scale surface roughness: (a) 2D and (b) 3D Chebyshev particles, (c) 2D and (d) 3D Gaussian random spheres (GRS), and (e) Chebyshev/GRS hybrid particle.

frozen (a phenomenon known as "riming") are typical exhibits of particles with small-scale surface roughness.

One of the first model geometries that was employed for studying the effects of small-scale surface roughness is a simple axisymmetric Chebyshev particle defined by the surface parameterisation

$$
r(\theta)=r_{0}(1+\epsilon \cos \ell \theta) .
$$

Here $r_{0}$ is the radius of the unperturbed sphere, $\epsilon$ is the deformation parameter (where $-1 \leq \epsilon \leq 1$ ), $\ell$ is the order of the Chebyshev polynomial, and $\theta$ is the polar angle in a spherical coordinate system with the origin in the centre of the sphere. Fig. 1 a shows a Chebyshev particle of order $\ell=44$.

Chebyshev particles of low orders have previously been used as a model for non-convex geometries in light scattering studies [34, 43, 44, 35]. One of the first studies of high-order Chebyshev particles [37] revealed a number of interesting facts about small-scale surface roughness. As one would expect, the optical properties of such particles change with $\ell$, at least for low values of $\ell$. However, as the order of the Chebyshev polynomial grows, the optical properties converge and no longer change with any further increase in $\ell$. Indication of the same convergence was found independently by [45] using spheres deformed by single-degree spherical harmonics.

Based on this observation, the following definition of small-scale surface roughness has been proposed in [46]: Suppose the particle surface is perturbed with a characteristic length scale $\Lambda$ and a characteristic perturbation amplitude $A$. (For example, for a Chebyshev particle, $\Lambda=2 \pi r_{0} / \ell$ and $A=\epsilon r_{0}$.) Then the surface perturbation is called a small-scale surface roughness if all of the following criteria are satisfied: 
1. The length scale $\Lambda$ is sufficiently small so that any further decrease in $\Lambda$ has no effect on the optical properties.

2. $\Lambda \ll 2 \pi r_{0}$, where $r_{0}$ is the characteristic size of the particle, so $2 \pi r_{0}$ is the characteristic circumference of the particle.

3. $\Lambda \ll \lambda$, where $\lambda$ is the wavelength of light.

4. $A \ll r_{0}$.

5. $A \ll \lambda$.

6. $A$ is sufficiently large so that the optical properties of the particle with a perturbed boundary surface differ from those of the corresponding unperturbed geometry.

The last criterion excludes purely geometrical surface perturbations that are so tiny that they have no noticeable effects on the optical properties.

Another finding in [37] was that the significance of small-scale surface roughness shows a rather peculiar dependence on the refractive index. For convex geometries, such as spheres and spheroids, it is well-known that the differences in the phase function of spheres and spheroids decrease and eventually disappear as the imaginary part of the refractive index is increased (e.g. Figs. 10.15 and 10.16 in [11]). However, high-order Chebyshev particles behave in the opposite way [37]; the phase function of high-order Chebyshev particles deviates more strongly from that of size-equivalent spheres for high values of the imaginary part of the refractive index.

The development of improved computational methods [47] made it possible to study the effect of small-scale surface roughness on particles with varying size. For axisymmetric, high-order Chebyshev particles with a refractive index typical for hematite aerosols at visible wavelengths, it was found that differences between spheres and spheroids disappear, while those between spheres and Chebyshev particles increase as the size parameter of the particles is increased [46].

These observations can be interpreted as follows. The light scattering properties of convex particles with smooth boundary surfaces, such as spheres or spheroids, are predominantly determined by the internal field inside the particle. At each point, this internal field becomes the source of partial fields which superimpose to form the scattered field. A diffeomorphic deformation of a sphere into a spheroid will cause a corresponding change in the internal fields, which will give rise to changes in the scattered field. However, as the absorption efficiency of the particle is increased (either by increasing the size parameter or the imaginary part of the refractive index), the internal field 
is quenched, and differences among the scattered fields of particles such as spheres and spheroids decrease. At the same time, the relative importance of induced surface currents on the scattered field increases. These, in turn, can become strongly modulated by the presence of small-scale surface roughness.

Similar investigation were repeated for refractive indices typical for ice particles at a far infrared wavelength of $47 \mu \mathrm{m}$ [46], as well as for mineral dust at visible wavelengths [48]. A general trend is that the impact of smallscale surface roughness on optical properties increases with the real part of the refractive index. This supports the interpretation given above, as the impact of surface currents on the light scattering behaviour is higher for optically hard materials.

While the extinction efficiency tends to be quite insensitive to smallscale surface perturbations, most other optical properties can be affected. For instance, for large hematite particles, the backscattering cross section of spheres can be up to 5 times larger than that of size-equivalent Chebyshev particles [47]. In the same study, the single scattering albedo of spheres was found to be higher by 0.08 , while the asymmetry parameter was lower by 0.13 .

These examples illustrate that one can learn much about complex morphological features, such as small-scale surface roughness, just by studying a simple axisymmetric model particle. To go any further requires the use of more elaborate models. As an example, a recent comparison employed 2D (axisymmetric) and 3D Chebyshev particles, as well as 2D and 3D Gaussian random spheres [48]. These are shown in Fig. 1 a-d. A 3D Chebyshev particle has the surface parameterisation

$$
r(\theta, \phi)=r_{0}(1+\epsilon \cos \ell \theta \cos \ell \phi)
$$

where $\phi$ denotes the azimuth angle in the spherical coordinate system. A Gaussian random sphere (GRS) is a particle with a spherical base geometry of radius $r_{0}$ that is stochastically deformed based on a prescribed covariance function of radius. The covariance function can be specified, e.g., by an angular correlation $\Gamma$ and a standard deviation $\sigma$, which are linked with the horizontal and vertical extent of the deformation scale (see [49] for details). In terms of our roughness lengthscale $\Lambda$ and roughness amplitude $A$, we have $\Lambda=\Gamma r_{0}$ (if $\Gamma$ is given in radians), and $A=\sigma r_{0}$. The goal of this study was to compare $2 \mathrm{D}$ and $3 \mathrm{D}$ roughness models, as well as regular and stochastic roughness models. 
It was found [48] that, for comparable values of $\Lambda$ and $\sigma, 2 \mathrm{D}$ roughness models yield optical properties that deviate more from the optical properties of unperturbed spheres than 3D roughness models. Regular and stochastic models for small-scale surface roughness have very similar effects on most except one optical property. The Mueller matrix element $F_{22}$ is identically equal to the phase function $F_{11}$ for unperturbed spheres. This does not change for particles with regular surface perturbations (2D and 3D Chebyshev particles), even though all other Mueller matrix elements can change appreciably (depending, as mentioned above, on size and refractive index). This means that the degree of linear depolarisation

$$
\delta_{L}(\Theta)=\frac{F_{11}(\Theta)-F_{22}(\Theta)}{F_{11}(\Theta)+F_{22}(\Theta)}
$$

is zero for spheres and Chebyshev particles (where $\Theta$ denotes the scattering angle). This is, at least, true for the relatively small perturbation amplitudes that have been studied so far. However, for 2D and 3D GRS, $\delta_{L}$ differs from zero even for small perturbation amplitudes, and it can vary among different stochastic realisations of the GRS geometry.

A major disadvantage of 3D GRS particles is the absence of any particle symmetries, which results in high CPU time requirements in light-scattering computations. A model which incorporates stochastic surface perturbations, while maintaining a high degree of symmetry is a Chebyshev/GRS hybrid particle shown in Fig. 1 e. The boundary surface of this particle is perturbed with a 2D Gaussian random roughness in the polar direction, and with a regular Chebyshev polynomial in the azimuthal direction. Thus, the degree of rotational symmetry of this particle is given by the order of the Chebyshev polynomial. Although this geometry has been implemented into the publicly available T-matrix program Tsym [50], no computational tests have been reported yet.

Further refinements of these models can be achieved by replacing the sphere with a nonspherical base geometry and perturbing its boundary surface with different roughness models. In [37] spheroids and infinite circular cylinders with high-order 2D-Chebyshev perturbation have been considered. Even more realistic model geometries have been employed; in [51], different Gaussian random base geometries with low correlation angle were used, and the surfaces of those were perturbed by a randomized surface roughness with varying roughness amplitudes. With such highly realistic geometries 
one starts to approach what we referred to in Sect 2.2 as "numerical laboratories". By systematically varying the surface roughness and size parameter, one can perform controlled numerical experiments with particle morphologies that are very close to real-world dust or ice particles, and study the effects on the optical properties [51].

The main practical limitations for studying particles with small-scale surface roughness come from numerical restrictions. Most studies reviewed above, with the exception of [51], were performed by use of Waterman's T-matrix method [52]. Despite recent progress in T-matrix calculations for particles with small-scale surface roughness [47], the method is still limited to small values of the perturbation amplitude $A$. Also, the method is most efficient for particles that have at least some degree of symmetry $[53,54]$. For irregular particles one can employ the discrete dipole approximation (DDA), which is often numerically more stable then the T-matrix method (e.g. [55]), so it is not limited to small perturbation amplitudes. However, it is also much slower. The DDA is a volume-integral equation method based on discretising the particle volume. The method becomes exceedingly slow in applications that require a grid consisting of a large number of cells. This is the case for particles with large size parameters, or geometries with fine morphological details, such as small-scale surface roughness [48]. These numerical obstacles are the primary factors that limit our present understanding of the impact of non-convex, sub-wavelength surface perturbations on optical properties of particles.

A very different model geometry is obtained by "dusting" a spherical particle with a large number of much smaller spheres [56, 57]. For such a geometry, light-scattering computations can be performed with the superposition T-matrix method [58]. It is possible that such a geometry is capable of mimicking the effect of realistic small-scale surface roughness.

\subsection{Overall nonsphericity}

One of the most fundamental factors in defining a particle's single-scattering properties is its overall shape. At the most fundamental level is the distinction between spherical and non-spherical particles. In general, scattering by spherical particles is very different from that by non-spherical particles. On the one hand, spherical particles have a very high degree of symmetry, resulting in strong, well-defined interference patterns, which can be sensitive to even smallest changes in the sphere radius, refractive index or the incident wavelength through their impact on phase. On the other hand, the 
spherical shape excludes any effects from particle orientation or variability in shapes, which are integral factors when considering populations of nonspherical particles. Size and composition can vary in both populations. One practical implication is that, for spherical particles, size integration should be carried out with a much finer resolution for convergent results. Especially for an ensemble of varying non-spherical particles, the size dependence of the single-scattering properties can be vastly weaker. To a lesser degree, this also influences the dependence on the refractive index, as illustrated by [59].

The dependence of the single-scattering properties on the overall particle shape for different non-spherical particles is, on the other hand, very complicated indeed. The simplest non-spherical particle shapes are the finite cylinder and spheroid, which both have one additional parameter to spheres to fully describe their geometry, related to their aspect ratio, and both possess axial symmetry. The single-scattering properties of spheroids have been extensively mapped and presented, for example, by [11]. As can be seen, different spheroidal shapes possess widely varying single-scattering properties. This fact gives shape distributions of spheroids great flexibility in mimicking scattering by different types of particles or their ensembles. Even scattering by cubical particles can be closely mimicked with a suitable distribution of spheroids [60]. This great flexibility is, however, a double edged sword, because it may allow good fits with incorrect physical parameters, such as with a wrong refractive index. Single spheroids, on the other hand, do not share the single-scattering properties of non-spheroidal particles with the same aspect ratios [61, 62], so one should not think that when using spheroids to mimic scattering by more complex particles, best results would be achieved using aspect ratios of the target particles for the spheroids. In addition, as a side effect, the shape distribution of spheroids that optimally mimics scattering by a certain target at one wavelength is no longer optimal at another wavelength [63].

The use of spheroidal model particles has reached a high degree of maturity. There exist ready-to-use look-up tables for optical properties of spheroids (e.g. [8, 64]) and ellipsoids [65]. Spheroids are being used for representing the optical properties of nonspherical aerosols in operational remote sensing retrieval methods. Such spheroid-based methods are employed for various ground- and space-based measurement programs and instruments, such as the Aerosol Robotc Network (AERONET) [66, 8], the Moderate Resolution Imaging Spectroradiometer (MODIS) [67], the Spinning Enhanced Visible and Infrared Imager (SEVIRI) [68, 69], and the Polarization and 
Anisotropy of Reflectances for Atmospheric Sciences coupled with Observations from a Lidar (PARASOL) research satellite [70]. The retrieval methods for these observations all employ the spheroid shape distribution described in [8], which has been tuned to fit the laboratory observations of light scattering by mineral dust reported in [71]. This spheroid model has also been used in the interpretation of lidar observations of mineral dust particles (e.g. $[72,73,74,75,76,77])$.

Similarly to spheroids, ellipsoids also possess great flexibility, and provide an even larger variety of shapes for fitting. Consequently, they can, potentially, provide even better fits to scattering patterns by different targets [78]. However, they also have a significantly lower degree of symmetry, which will strongly increase CPU-time requirements in light-scattering computations. Although the performance of ellipsoids have not been studied under different conditions, it is reasonable to expect that the peculiarities of spheroid fitting also apply to ellipsoids.

Another simple, non-spherical model geometry is the polyhedral prism. According to [79], the single-scattering properties of different symmetric polyhedra vary surprisingly little. When the number of corners in the polygonal cross section is increased, the single-scattering properties converge towards that of a circular cylinder, but even for a small number of corners (from four to seven in the study), the differences are fairly modest. Likewise, when the aspect ratio is changed, scattering remains almost unchanged. The probable cause is that the aspect ratio does not affect the angles between the particle faces but only the relative size of the faces. We will discuss pristine shapes in more detail in Sect. 3.3.

Asymmetric polyhedra have been considered by [80], who focused on convex hexahedra with quadrilateral faces. Their scattering properties differ clearly from those of symmetric polyhedra, which can be attributed to their lack of symmetry. Incidentally, the single-scattering properties were surprisingly similar to those of complex dust particles. Similarly to symmetric polyhedra, these model particles do not possess strong particle-to-particle variability in their single-scattering properties, albeit the varying angles between the faces.

The Gaussian random sphere geometry has also been used to investigate the impact of overall particle shape on scattering. For example, [81] consider Gaussian spheres where the $\sigma$ parameter is varied from 0.05 (almost spherical particles) to 0.2 (considerably non-spherical particles). They find that the increase in $\sigma$ makes the phase function more featureless, removing 
the interference structures associated to spherical particles, and reduces the backscattered intensity; the degree of linear polarization for unpolarised light becomes more positive and smooth; and the depolarization ratio generally increases with increasing $\sigma$. In this one-parameter-controlled increase of nonsphericity, depolarization acts as a reasonable measure for non-sphericity, but as discussed below, this may be an exception rather than a rule. In particular, [81] did not consider Gaussian spheres with different angular correlation, which might impact depolarization considerably. For example, [82] found that, for backscattering and small size parameters, highest depolarizations were obtained with correlation lengths that produced elliptical deformation, that is, non-sphericity in its largest scale.

Of particular interest in establishing how the overall shape impacts scattering is the study by [83], where single-scattering properties for 15 different model types were investigated, including spheres, sphere clusters, spheroids, polyhedra, and several types of irregular shapes. For each non-spherical particle type, ensemble and orientation-averaged scattering properties were considered, so the comparison did not address how individual shapes impact scattering but rather what is typical scattering for different morphological types. The key findings of the study can be summarized as follows:

- Backscattering was generally weaker for non-spherical than for spherical particles.

- The degree of linear polarization for scattering of unpolarised incident light turned out to be the most sensitive scattering matrix element to particle morphology of those analysed.

- In some cases, similarly looking morphologies could have distinctly different single-scattering properties, while clearly different morphologies could lead to very similar scattering.

- High-aspect ratio particles scattered differently to more equidimensional morphologies: depolarization characteristics were clearly different, and highly elongated particles generally had higher asymmetry parameters.

- The dependence of depolarization on particle morphology defied simplistic explanations: depolarization is a useful indicator for anisotropy (due to non-spherical shape, for example), but not a measure of anisotropy. 
- Some particle types which had seemingly very complex shapes, had nevertheless distinctive interference structures in their scattering patterns, while other similar particle types did not. Those that did had spherical base shapes, suggesting that complex particle geometries perhaps should not be modelled using deformed spheres.

- Ensembles of different non-spherical particles scattered surprisingly similarly, suggesting that scattering by one type of model particles can be mimicked by another, which may be seen either as a curse or blessing. It is emphasized that this finding does not implicate similarities in scattering between different, individual non-spherical particles.

- Some morphological characteristics, such as surface roughness or smallscale internal structure, might have a systematic impact on scattering characteristics.

Regarding the effect of ensemble averaging, it appears that for certain regular shape classes such as spheroids and ellipsoids, differently shaped members can have distinctly different single-scattering properties, while some other morphologies, such as different polyhedra, show much weaker particleto-particle variation. Indeed, the distorted Koch fractals considered by [84], which are very complex concave polyhedra, showed nearly non-existent variability among the random members of the model ensemble. We speculate that the key to changing substantially the single-scattering properties within a shape class is related to organized changes in the interference in the internal field. For very complex particles, the changes are not organized, and for particles with parallel faces, for example, the changes are simply too small.

\subsection{Pristine shapes}

Under tropospheric conditions, water ice usually crystallises in the hexagonal close-packed (hcp) crystal structure, which can give rise to ice prisms with hexagonal cross section, as well as various snow-flake shapes with hexagonal base geometry. Pure sodium chloride has face-centred cubic (fcc) crystal structure, which, under ideal conditions, gives rise to cubic salt crystals. Particles of such pristine shapes give rise to distinct optical phenomena that can be observed in nature, such as haloes caused by atmospheric ice crystals [85]. While many common halo phenomena may arise from crystals that are only partially pristine, some rare forms (e.g., Tricker arc and subparhelic 
circle) require very complex and specific light paths with multiple internal reflections that are unlikely to be possible except in fully pristine crystals.

In the real world these halo forms are rare. More often than not, the conditions are not ideal, particle formation processes can be rather complex, and both ice particles and salt aerosols can contain impurities. As a result, irregular ice and salt particles usually dominate over pristine shapes (e.g. $[86,87])$. In spite of that, the use of pristine geometries in light scattering computations can be useful in various contexts. For instance, the use of pristine ice crystal shapes can serve as a first step in studying the impact of ice particle morphology on optical properties; such studies can be conducted by comparing computational results for idealised hexagonal ice crystals to actual measurements (e.g. [88]) or to corresponding computational results obtained for more complex, irregular ice crystal models (e.g. [89, 90]). More elaborate pristine model particles, such as stellar crystals and solid or hollow bullets [91], and fern-like or dendritic crystals [92] have also been used.

At the turn of the millennium, idealised shapes were employed in the earliest attempts to go beyond the use of axisymmetric model particles. One of the first tests of the applicability of spheroidal model particles to mineral dust and other non-spherical aerosols considered finite prisms with cross sections in the form of regular polygons with $N$ corners, where $N$ was varied between 4-8 [93]. The idea of that study was based on the notion that prisms have more complex shapes than spheroids, since they have a lower degree of symmetry. The question was if one could reproduce the optical properties of less symmetric particles with a suitable ensemble of more symmetric, i.e. simpler model particles. This was tested by fitting the optical properties of prisms by optimising the shape-distribution of spheroids. It was found that spheroids were, indeed, able to reproduce the optical properties of the ensemble of prisms. However, later comparisons of prisms and spheroids to measured Mueller matrices of feldspar aerosols showed that spheroids are more versatile model particles than prisms, since they cover a larger range of Mueller matrices than prisms as one varies their aspect ratio [79]. This showed that more complex (i.e. less symmetric) geometries are not automatically more versatile model particles for reproducing optical properties of realistic, non-spherical aerosols.

In another study double-pyramids and other multi-faceted concave and convex geometries were employed as model particles, and the computational results were compared to microwave analogue experiments [94]. A suitable mixture of concave and convex model particles was able to reproduce the 
measured phase function.

The use of pristine model particles can also be an option if computational limitations prohibit the use of more complex model particles. This can be the case in climate modelling or remote sensing inversion schemes. For instance, hexagonal ice prisms have been used to generate optics look-up tables for inversion schemes of remote sensing observations of ice clouds at IR wavelengths [95]. On the one hand, the use of hexagonal prisms goes significantly beyond simpler models based on spheres or finite circular cylinders. On the other hand, more complex, irregular model particles would have required too much computer time for generating an optics look-up table.

Pristine model geometries can also be used if, due to insufficient data on the particles to be modelled, there is no motivation for employing more sophisticated model geometries. For example, very little is known about the shape of realistic dried sea salt aerosols, but they rarely seem to have perfect cubic shape [87], as they are not composed of pure sodium chloride crystals. Rather, they contain a small fraction of other elements, such as potassium, calcium, magnesium, and sulphur, and they also mix with sulphate or organic substances originating from biogenic marine precursors. In the absence of detailed information on the particle shape, a cubic particle model can be used as a reasonable first-guess geometry in light-scattering computations [96].

\subsection{Aggregates}

Aggregation of smaller particles into larger clusters results in particles with often irregular shape and with complex topological properties. One important class of aggregates are light absorbing carbon (LAC) aerosols, which are composed of small amorphous carbon spherules (monomers). The monomers typically have radii in the size range of 10-25 nm, and the aggregate sizes range from the Aitken to the accumulation mode [97]. Fly

ash particles, which typically consist of the inorganic fraction (e.g. clays) of coal combustion particles, are forming aggregates that have shapes similar to LAC, but the fly ash aggregates can cover a very large size range from the Aitken to the coarse mode [98]. Ice cloud particles can form regular rosettes composed of hexagonal ice bullets, or irregular aggregates composed of ice columns (e.g. [99]). Ice aggregates are most frequent for particles with maximum dimensions above $100 \mu \mathrm{m}$. Aggregates composed of silicate monomers are believed to be an important fraction of cosmic dust and planetary regoliths (e.g. $[2,100])$. 
Historically, light scattering modelling of aggregates proceeded in a slightly different way than that of other particle morphologies. Rather than introducing radical simplifications to the particle morphology, many of the first studies used quite realistic morphological models, but they drastically simplified the theoretical description of the electromagnetic scattering problem. One approach is to assume that the monomers are sufficiently small so that they can be treated by Rayleigh scattering. The optical properties of the aggregate can then be computed with the discrete dipole approximation [101]. Another approach is to invoke the Rayleigh-Debye-Gans (RDG) approximation $[102,103]$. The RDG approximation neglects electromagnetic interaction among the monomers in the aggregate. In parallel, rigorous solutions of the electromagnetic scattering problem for aggregates of touching spheres have been developed for computing the extinction, scattering, and absorption cross sections [104] as well as the Mueller matrix [58]. Implementations of these rigorous methods are publicly available $[105,106]$. The method in [105] also includes an approximate approach based on limiting electromagnetic interaction among the monomers to an adjustable interaction radius.

In several applications approximate methods have been demonstrated to give sufficiently accurate optical properties (e.g. [107]). However, a recent comparison of approximate calculations based on [105] with those based on rigorous theory for LAC aggregates revealed that neglecting or limiting electromagnetic interaction among the monomers can introduce significant errors in the absorption and scattering cross sections [108]. With the availability of more computational power and of parallelised computer codes [106] the use of approximate methods has steadily declined, and an increasing number of studies based on the numerically exact superposition T-matrix method has been reported $[109,110,111,112,113,114,56,57,115]$.

The main problem in modelling the geometry of fractal aggregates arises from the fact that the variety of aggregate shapes is limitless. This calls for a statistical approach. The fractal structure of aggregates consisting of $N_{s}$ monomers of radius $a$ can be described by the scaling relation [116]

$$
N_{s}=k_{0}\left(\frac{R_{g}}{a}\right)^{D_{f}}
$$

where $D_{f}$ is the fractal dimension, $k_{0}$ is the structural prefactor, and

$$
R_{g}=\sqrt{\frac{1}{N_{s}} \sum_{i=1}^{N_{s}} r_{i}^{2}}
$$



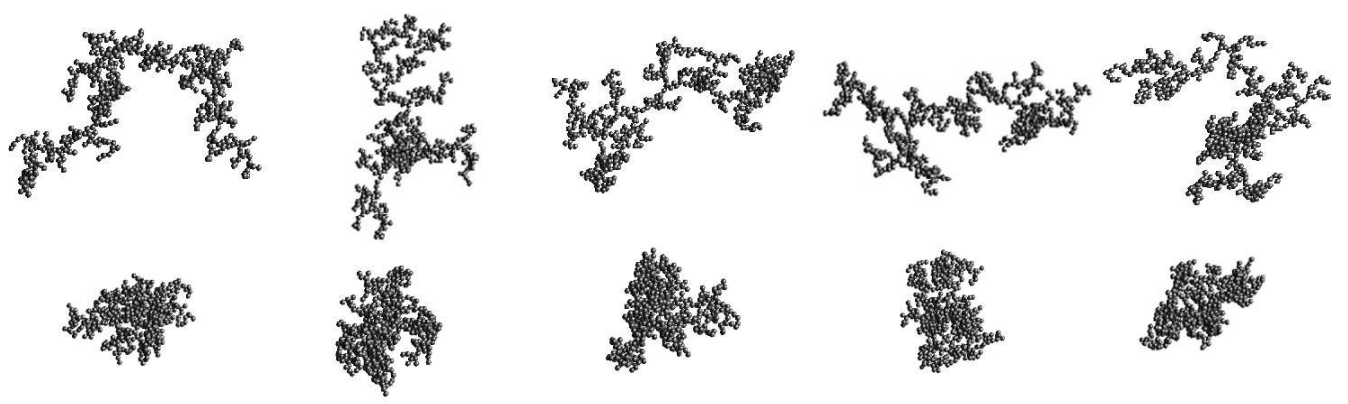

Figure 2: Aggregates that satisfy the scaling relation in Eq. (4), each consisting of $N_{s}=600$ monomers. Top row: $D_{f}=1.8, k_{0}=1.3$; bottom row: $D_{f}=2.4, k_{0}=0.7$. Each row shows five stochastic realisations belonging to the same class of fractal aggregates.

denotes the radius of gyration, where $r_{i}$ is the distance of the $i$ th monomer from the aggregate's centre of mass. The main approximation is that all monomers are assumed to have the same radius, while in reality, the monomer sizes can vary within an aggregate. Also, the spheres are assumed to be only touching, not overlapping or connected by bridges ("sintering").

The scaling relation given in Eq. (4) provides a convenient method for classifying a virtually infinite variety of aggregate shapes by labelling them with the set of parameters $\left\{N_{s}, a, D_{f}, k_{0}\right\}$. Figure 2 illustrates the variability of aggregate geometries for a given set of fractal parameters. Realistic particles formed in diffusion-limited aggregation processes are known to obey Eq. (4) (e.g. [116]). However, this classification is only useful for our purposes if it provides us with "optical equivalence classes", i.e., if all particles belonging to the same class $\left\{N_{s}, a, D_{f}, k_{0}\right\}$ have nearly identical optical properties. Remarkably enough, calculations for different stochastic realisation of LAC aggregates belonging to the same class $\left\{N_{s}, a, D_{f}, k_{0}\right\}$ confirm that these particles have essentially identical optical cross sections [113]. For differential scattering properties, such as the backscattering cross section, the variation among different fractal aggregates having the same fractal parameters can be somewhat larger [113].

Aggregate model geometries for light scattering calculations, such as those in Fig. 2, have been generated with algorithms [104] that sequentially build up clusters of spheres by randomly attaching new spheres, under the constraint that in each step of the build-up process the cluster needs to satisfy Eq. (4). Thus the scaling relation holds for every size scale of such a clus- 
ter, as it must for a fractal geometry. With such model particles, one can conduct controlled numerical experiments to study the dependence of the optical properties on the physical and dielectric properties of the aggregates. In some of the earlier studies of LAC aggregates, $D_{f}, k_{0}, a$, and the real and imaginary parts of the refractive index, $m=n+\mathrm{i} \kappa$, have been varied over very large ranges. For instance, a variation of $D_{f}$ in the range between 1 and 3 reveals a strong dependence of the optical cross sections and the asymmetry parameter on the fractal dimension [111]. Also, varying the monomer radius $a$ in the range between $5 \mathrm{~nm}$ and $60 \mathrm{~nm}$ reveals a high sensitivity of the scattering cross section, single scattering albedo, and asymmetry parameter [109]. Refractive index values that have been used in earlier studies cover the range from $1.25+0.25 \mathrm{i}$ up to $2+\mathrm{i}$ (see [117] and references therein). However, more recent measurements have allowed us to significantly reduce the range of uncertainty in fractal parameters, monomer radii, and refractive index values. Laboratory-generated LAC tend to have fractal parameters around $D_{f}=1.8$ and $k_{0}=1.3$ [97]. Recently improved electron tomography techniques [118] applied in field observations [119] yield median values of $D_{f}=2.2$ and $k_{0}=0.7$ near emission sources and $D_{f}=2.4$ and $k_{0}=0.7$ for long-range transported LAC that has not mixed internally with other chemical species. A review of existing measurements in conjunction with theoretical models allows to confine refractive index values to a range between $m=1.75+0.63 \mathrm{i}$ and $m=1.95+0.79 \mathrm{i}[97]$. The monomer radius of LAC typically varies in the range of $a=10 \mathrm{~nm}$ and $25 \mathrm{~nm}$. Field observations show a mean value around $22 \mathrm{~nm}$ [120].

These recent observational constraints allow us to perform more focused sensitivity studies. In [114] a comparison was conducted of a lacy and a compact aggregated model with $\left(D_{f}, k_{0}\right)=(1.8,1.3)$ and $\left(D_{f}, k_{0}\right)=(2.4,0.7)$, respectively, (see Fig. 2); both single-scattering computations and radiative transfer simulations were performed. The differences in optical properties and radiative forcing rates between the two aggregate models was significantly smaller than those between the aggregates and a homogeneous sphere model. This suggests that a variation of the fractal parameters within realistic limits is a minor source of uncertainty in radiative transfer calculations.

Another sensitivity study [108] suggests that a variation in the monomer radius $a$ between $10-25 \mathrm{~nm}$ has little impact on the optical cross sections, but it does affect the single scattering albedo. The best agreement with reported observations (see [97] and references therein) is obtained for $a=25 \mathrm{~nm}$ [108], which is consistent with recent field observations [120]. 
These examples illustrate how realistic aggregate models in conjunction with numerically exact T-matrix computations can be used as what we call a "numerical laboratory". Curiously, these rigorous numerical calculations for highly realistic model particles still underestimate observed mass absorption cross sections (MAC) of pure LAC aggregates [108]. A likely, and surprising, explanation is that the model particles are not yet sufficiently realistic. We mentioned that the fractal aggregate models assume a constant monomer radius $a$, but we also mentioned that the optical cross sections are not very sensitive to a variation in $a$. The assumption of a constant $a$ is, therefore, not a likely cause of the underestimation of MAC by the model calculations. A more severe approximation may be the touching-sphere assumption. Modelling results for sintered and non-sintered titanium dioxide aggregates shows that sintering can significantly increase the extinction cross section in the UV and visible spectral range [121]. Similar studies on soot have been performed [122], but not for aggregates that are typical for atmospheric LAC. Thus it is still an undecided question if sintering has an important impact on the absorption cross section of LAC aggregates in the atmosphere. Another simplification in the studies cited above is to assume bare aggregates. Under realistic conditions, LAC aggregates originating from combustion processes are often covered by a layer of organic substances, which could very well enhance the absorption cross section. This illustrates that even model particles with very complex geometries that are believed to be highly realistic can contain unsuspected simplifications; very subtle morphological features, such as sintering or thin coatings, may have a significant impact on aerosol optics. However, further studies are needed to corroborate these suspicions.

The study of the optical properties of LAC aggregates has matured to the point that aerosol optics look-up tables based on numerically exact T-matrix computations have recently been created and implemented in a chemical transport model [112]. It may be surprising that one can afford the use of model particles as complex as those shown in Fig. 2 in environmental modelling. There are, essentially, two factors that make the task of creating look-up tables much simpler for LAC than for other types of aerosols, such as mineral dust. One factor is that pure LAC aggregates are mostly limited to the Aitken and accumulation size range, while mineral dust particles can be much larger. The second factor is that the optical properties of strongly absorbing LAC particles are, by contrast to weakly absorbing aerosols, very smooth functions of size and wavelength (see, e.g., Fig. 5 in [112]). Thus, to compute size-averaged optical properties one only needs to perform com- 
putations for a limited number of discrete sizes [112]. By contrast, optical properties of weakly absorbing particles can strongly oscillate with size and wavelength. For instance, to resolve the oscillations in backscattering cross sections of weakly absorbing spherical particles, one would need to use a size resolution of $k \Delta r=0.025$ [64], where $k=2 \pi / \lambda$ is the wavenumber, and $\Delta r$ is the size resolution. For spheroids, due to orientation averaging, the required resolution is reduced to $k \Delta r=0.2$ [64]. Thus, depending on the wavelength, the number of particle sizes required for generating look-up tables for weakly absorbing particles is on the order of $10^{3}-10^{4}$. For highly absorbing particles, it is sufficient to perform calculations for $\sim 10^{1}$ discrete sizes.

\subsection{Inhomogeneity}

\subsubsection{Encapsulated aggregates}

The absorption cross section of LAC aerosols can be significantly enhanced due to mixing with other aerosol components [123, 124]. For this reason, the mixing state of $\mathrm{LAC}$ is of considerable interest in climate modelling. As LAC aggregates age in the atmosphere, they get oxidised and become more hydrophilic. As a result of condensation processes, they become partially or fully covered by liquid-phase materials, such as secondary inorganic compounds [125], organic substances [126], salt [127], and water [128]. The resulting geometry is an LAC aggregate encapsulated in a shell of other aerosol compounds that are weakly absorbing at UV and visible wavelengths. The optical properties of these particles are strongly influenced by the high optical contrast between the LAC core and the shell, which persists over a large part of the solar spectrum. At UV and visible wavelengths, the imaginary part of the refractive index of LAC [129] is substantially higher than that of, e.g., sulphate [130]; at IR wavelengths, the difference decreases, since sulphate becomes more strongly absorbing. On the other hand, the real part of the refractive index of LAC [129] steadily increases from the UV to the IR, while that of sulphate [130] slowly decreases. (See also Fig. 3 in [41].)

In environmental modelling, one frequently neglects internal mixing; one simply treats LAC and weakly absorbing aerosol components as externally mixed (e.g. [131]), i.e., as physically separated particles, as illustrated in Fig. 3 b. A slightly more sophisticated approach is to treat LAC and weakly absorbing material as a homogeneous internal mixture [132], as shown in Fig. 3 c. The mixture is characterised by an effective refractive index, which is 

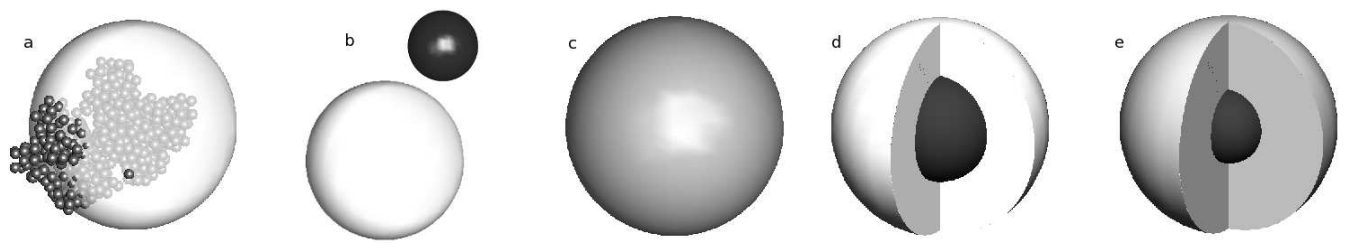

Figure 3: Models for encapsulated LAC: (a) encapsulated fractal aggregate, (b) externally mixed homogeneous spheres, (c) internally mixed homogeneous sphere/EMA, (d) coreshell, and (e) core-grey shell.

computed from the refractive indices of LAC and of the other weakly absorbing components by use of effective medium approximations (EMA) [133]. In either case, the particles are usually assumed to have spherical shape. The simplest morphological model that attempts to mimic the inhomogeneous morphology is a concentric core-shell model with an LAC core coated with a shell of weakly absorbing material [134], see Fig. 3 d. Extensions of this model are a non-concentric core-shell model [135], a dimer consisting of an LAC sphere attached to the surface of a sphere of weakly or non-absorbing material [117, 136], and a dimer of concentric core-shell particles [117].

For instance, it was found [135] that an LAC core encapsulated in a shell of sulphate is mostly sensitive to the LAC volume fraction (i.e. to the relative sizes of LAC and sulphate), and much less sensitive to the position of the LAC inclusion inside the sulphate sphere. More extensive calculations later confirmed that concentric core-shell models yield absorption cross sections that are similar to those computed with off-centre core-shell models, as long as the LAC cores are not much smaller than those typically encountered in atmospheric aerosols [123]. However, for small LAC inclusions encapsulated in large water cloud droplets, the absorption of electromagnetic energy is larger for centred than for off-centre LAC inclusions [135].

Comparisons of homogeneous spheres/EMA models with core-shell model calculations (where the shell was assumed to be water) demonstrated that the model agreement is best for LAC cores with small size parameters [132]. A later study replaced the simple core-shell particles with a model in which the LAC is distributed more randomly within the host, and where the overall shape of the particle can vary from spherical to spheroidal [137]. Here the EMA model was found to overestimate absorption and to underestimate total and differential scattering cross sections.

Dimers of LAC spheres and weakly absorbing spheres $[117,136]$ as well 
as more sophisticated models consisting of LAC fractal aggregates attached to the surface of a sulphate, dust, or silica sphere [110] have been considered. Such models represent LAC aerosols that have mixed internally by coagulation with other aerosols. However, coagulation processes among Aitken and accumulation mode particles have extremely low rate constants; coagulation is a process that only contributes appreciably to scavenging of nucleation particles by accumulation mode particles. Thus, such coagulated geometries are extremely rare in the atmosphere. By far the dominating process that mixes LAC with other aerosol components is condensation, which gives rise to encapsulated geometries.

The studies cited above were mostly based on simple model geometries; they illustrate that we can gain a lot of insight from studying such simple particles and from observing the dependence of the optical properties on the parameters that characterise these model geometries. However, the investigative potential of highly simplified model geometries is sooner or later exhausted, and more realistic model geometries are required to make further progress. The construction of such models can be guided by the analysis of transmission electron micrographs of individual LAC aggregates encapsulated in a shell of sulphate [138], organic matter [139, 120], or sodium chloride [127].

For instance, one of the first studies that went beyond simple core-shell models considered two realisations of a sulphate shell with multiple spherical LAC inclusions [138]. The fractal aggregate structure of LAC was neglected by representing each LAC aggregate encapsulated inside the sulphate host by a size-equivalent homogeneous sphere. But the presence of multiple inclusions was accounted for. The total cross sections, single scattering albedo, and asymmetry parameter computed for these particles were compared to corresponding results obtained for simplified model geometries, such as homogeneous spheres with effective refractive index, sulphate-LAC-sulphate sandwich spheres, sulphate-LAC hemispherical spheres, sulphate-LAC coreshell, and LAC-sulphate core-shell spheres. The agreement between the simplified particle models and the more realistic models depended on the LAC volume fraction.

Subsequent studies accounted for even more geometrical details by representing the LAC particle by a fractal aggregate consisting of small carbonaceous monomers, which are encapsulated in a coating of weakly absorbing material, such as organic matter [120], sulphate [140, 41], or sodium chloride [127]. This model is illustrated in Fig. 3 a. For instance, accounting 
for the aggregate shape and the, generally, off-centre position of the aggregate within its encapsulating host particle was found to reduce the radiative forcing effect of LAC by $20 \%$ as compared to a simple concentric core-shell model [120].

Computational results obtained from the use of morphologically realistic encapsulated aggregates has called into question some long-held beliefs. Previously, it has been taken for granted that core-shell spheres are a more realistic model for encapsulated LAC aggregates than externally mixed spheres or a homogeneous sphere/EMA model (e.g. [141, 10]). Comparison with detailed calculations for encapsulated aggregate models showed that none of these simple particle models provides a realistic estimate of the optical properties of internally mixed LAC aerosols [140, 41]. While a homogeneous sphere model tends to overestimate absorption, a core-shell model and, even more so, an external mixture model underestimate the absorption cross section of encapsulated LAC aggregates. Even more importantly, these studies provided us with a physical understanding of the impact of particle morphology on aerosol optics by explaining the physical causes for the shortcomings of simplified model geometries [140,41]. In short, approximating LAC as a compact sphere, such as in the core-shell and external mixture models, underestimates absorption because the electromagnetic field cannot penetrate deeply into a homogeneous sphere, thus excluding much of the mass inside the LAC sphere from interacting with the field. In a realistic LAC aggregate the LAC mass is more spread out, so that less mass is shielded from interacting with the field. On the other hand, the homogeneous sphere/EMA model distributes the LAC mass too evenly, thus overestimating absorption.

Now we understand that the morphological property that makes the largest impact on the total optical scattering and absorption cross sections of these types of particles is how fluffily the LAC mass is distributed within the shell. We can exploit this insight to devise simplified model geometries that give a more accurate representation of optical properties. In a first attempt concentric 3-layered spheres were considered consisting of a sulphate core, an LAC inner shell, and a sulphate outer shell [140]. The total size and LAC volume fraction of these model particles was identical to that of the encapsulated aggregate particles, while the radius of the LAC shell was a free tuning parameter. By varying this parameter one can adjust the amount of LAC mass that interacts with the incident field. It was found that a suitably tuned model was able to accurately reproduce the total cross sections, single scattering albedo and asymmetry parameter, where the comparison was per- 
formed for one UV, one visible, and one near-IR wavelength [140]. However, this model is computationally too slow to be used in building aerosol optics look-up tables. For this reason, an even simpler "core-grey shell" model has been proposed [41], in which only part of the LAC mass resides in the spherical core, while the remaining LAC mass is homogeneously mixed with the concentric spherical sulphate shell (and the effective refractive index of the mixture is computed by EMA). This model is illustrated in Fig. 3 e. Again, the total particle volume and the LAC volume fraction are fixed, while the partitioning of LAC between the core and the "grey shell" is a free parameter of model, which allows us to adjust the amount of LAC mass that is interacting with the field. By performing detailed broadband calculations from the UV-C to the mid-IR and comparing the results to the encapsulated aggregate model, it was found that this model gives highly accurate results for not only the total cross sections, single scattering albedo and asymmetry parameter, but also for the backscattering cross section and absorption Angström exponent [41]. Thus this model is a promising candidate for use in both climate models and, conceivably, remote sensing applications. The one optical property it entirely fails to predict correctly is the degree of linear depolarisation $\delta_{L}$, which is identically zero for spherically symmetric particles. However, the model calculations for encapsulated aggregates indicate that realistic encapsulated LAC aggregates depolarise only weakly with $\delta_{L}$-values on the order of just a few percent.

This example illustrates how complex, morphologically realistic particle models can be put to use. They help us to gain a more thorough understanding of the physics of the scattering process, which can enable us to construct better simplified model geometries to be used in computationally demanding practical applications.

Finally, we point out that the importance of encapsulated aggregates is by no means limited to aerosols in the terrestrial atmosphere; they can also be important in astronomy. Silicate particles in interstellar space can aggregate and later be covered by a mantle of carbon [142] or of ices (e.g. $\mathrm{H}_{2} \mathrm{O}, \mathrm{CO}_{2}$, $\left.\mathrm{CO}, \mathrm{O}_{2}, \mathrm{~N}_{2}\right)[2]$.

\subsubsection{Porous particles}

By porosity we refer to material filled with internal cavities, containing gas

or vacuum. As such, it can be considered an extreme form of inhomogeneity. Vesicular volcanic ash is a prime example of such particles, but also many terrestrial ice particles, such as graupel, hail and ice crystals can have internal 

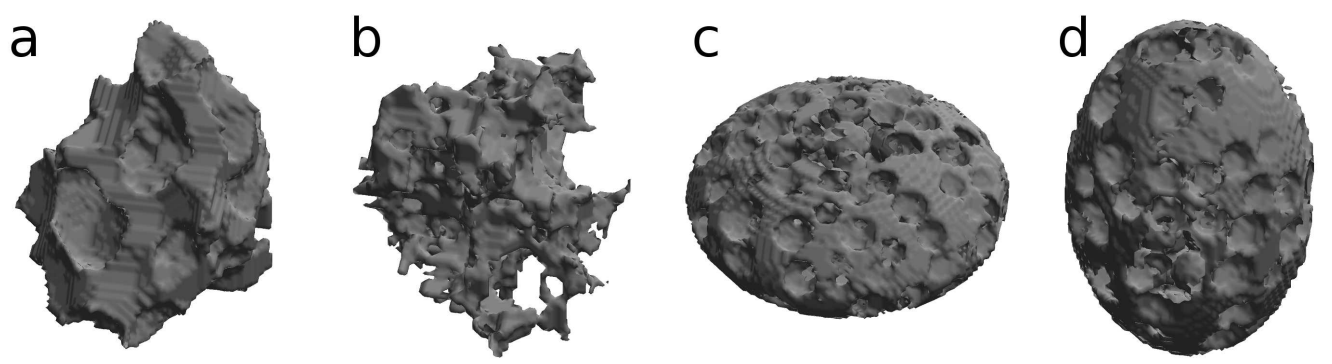

Figure 4: Models for porous particles: (a) volcanic ash with large vesicles [143], (b) volcanic ash with small vesicles [143], (c) porous oblate spheroid [60], and (d) porous prolate spheroid [60].

air pockets. Cometary dust may also include porous particles. Fig. 4 reviews selected models developed for porous particles.

Porosity has a potential to hugely impact the single-scattering properties of particles. Overall, the impact appears to bear similarities with that of surface roughness, tending to smooth out the phase function, decrease the backscattering cross section, and promote positive linear polarization with a bell-shaped angular dependence with the maximum in the side-scattering angles. For strongly depolarizing host particles, it appears to weaken the depolarization [143], which may be linked to reducing the effective refractive index. The effect does not only depend on the amount of porosity, but also on the size of the internal cavities, such that fewer and larger porous cavities produce a weaker effect than more but smaller cavities that have the same degree of porosity. The impact on the asymmetry parameter $g$ appears to be non-monotonic, while the single-scattering albedo $\varpi$ is increased. The latter is an expected result of reducing the amount of material where absorption can take place. It is also possible that the porous cavities scatter some of the radiation penetrating into the particle back to the particle surface.

In the thermal infrared, neither mass- nor volume-equivalent spherical model particles accurately reproduce the optical properties of more realistic porous models of volcanic ash particles [144]. This impacts the brightness temperature difference $(\mathrm{dBT})$ that is predicted by either particle model. It is estimated that spherical model particles can introduce errors in ash mass retrievals based on dBT observations from Earth-viewing satellites on the order of some tens of percent [144]. 
For strongly absorbing particles, porosity appears to profoundly change the size dependence of the single-scattering properties [145]. For example, the maximum degree of linear polarization and depolarization ratio, which show a clear minimum and maximum, respectively, when the particle diameter is about one wavelength for compact, absorbing particles, show clear decreasing and increasing trends with increasing size, respectively, for corresponding porous particles.

A recent study [60] addressed, among other things, the question whether light scattering by porous particles can be mimicked by homogeneous model particles. The optical properties of ensembles of silicate particles of different shapes, including Gaussian random spheres, cubes, and prolate and oblate porous spheroids, were computed. Then it was attempted to fit the simulated Mueller matrices with a suitable shape-distribution of size-equivalent homogeneous spheroids. The fitting was done by adjusting the shape-distribution of the spheroids. For the porous silicate spheroids the fitting was performed first with mass-equivalent homogeneous spheroids having a refractive equal to those of the porous spheroids, and second with volume-equivalent homogeneous spheroids having an effective refractive index of the silicate/vacuum mixture. Remarkably enough, the fitting exercise was successful for all but the porous particles, despite the fact that the porous spheroids outwardly bear the closest resemblance to the homogeneous spheroids. This indicates that porosity, perhaps even inhomogeneity in general, is a very fundamental morphological property that defies any attempts to mimic its impact on scattering by use of simple homogeneous model particles. We mentioned earlier that the scattered field can be pictured as the result of superimposing partial waves originating from different points inside the particle. Inhomogeneity entirely changes the topology of the particle, thus of the internal field. It appears that this effect is very difficult to reproduce with simple, homogeneous model particles, and that, therefore, porosity should be explicitly accounted for when computing single-scattering properties, unless porous cavities are only present in very minor quantities.

When porosity occurs on a size scale sufficiently smaller than the wavelength, it may be possible to account for it using effective medium approximations, but there appear to be no studies to prove this. A suitable surface roughness model might also be able to mimic many of the features with suitable parameters but, again, explicit proof is lacking, especially on the consistency of such a modelling approach. It may well be that the roughness parameters would have to be changed as a function of size parameter, for 
example. This is certainly an area where more research would be welcome.

\subsubsection{Compact, inhomogeneous particles}

By definition, inhomogeneous particles are macroscopically composed of more than one material: the refractive index of the particle changes internally. Inhomogeneity is a very common feature among atmospheric particles: already the formation of an aerosol particle through nucleation is likely to be a heterogeneous event and, while ageing in the atmosphere, the particles often mix with other aerosol particles or collect a surface layer of another material. Inhomogeneity scales vary from nearly molecular-level mixing to larger-scale coatings and agglomerated particles, where the different materials are clearly distinct. Effective-medium approximations of the material refractive index are often used to represent inhomogeneity. These approximations should not, however, be carelessly used outside their range of validity where the scale and the internal distribution of inhomogeneity are especially significant.

Despite being a common feature, inhomogeneity has been given notably little attention in light scattering considerations, at least compared to the number of studies made on the impact of particle shape on scattering. This is, at least partially, a consequence of the lack of measured data on the internal structure of atmospheric particles, but probably even more due to the fact that rigorous methods and models for solving light scattering by irregularly shaped inhomogeneous particles have been implemented only recently. Only in the special cases of a coated sphere and a multilayered sphere can an analytical solution be obtained as an extension to the Mie theory; otherwise, the methods for inhomogeneous, wavelength-scale particles are mostly volume-integral methods where scattering modelling may become very timeconsuming as, in a general case, one cannot take advantage of any symmetries. Therefore, it is once more a question of choosing the balance in between the possible and the sensible solutions. For example, a coated sphere can be considered as the simplest case of an inhomogeneous model particle, and yet it has proven to be a reasonably well-performing model even for a complex particle composed of a light absorbing carbon particle partly inside a soluble shell [41], only requiring that the volume fractions and the material refractive indices are appropriately chosen to correctly account for absorption. The choice of an appropriate modelling scenario becomes even more difficult if the particles are much larger than the wavelength, because arbitrary inhomogeneity cannot be considered by any available ray optics method. Some 

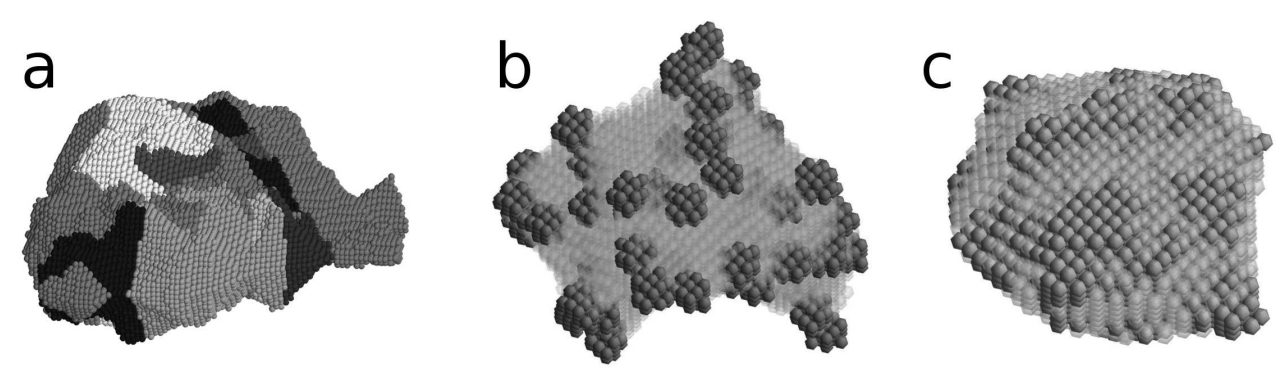

Figure 5: Models for inhomogeneous particles: (a) Stereogrammetrically retrieved mineral dust particles [62], (b) concave-hull-transformed aggregates of spheres [147], and (c) concave-hull-transformed Gaussian random spheres [147].

approaches, however, have incorporated small, diffuse internal scatterers that may in some cases be interpreted to mimic internal structure and inhomogeneity $[146,89]$.

Inhomogeneity is particularly important to consider in the context of mineral dust particles because of the great abundance of different mineralogical species. Then again, the differences in the refractive indices of the minerals are not necessarily large and, therefore, the impact of inhomogeneity on scattering can remain quite modest, as was noticed by [62] in their study where the inhomogeneity of four mineral dust particles was faithfully reproduced to volume-discretised, stereogrammetrically retrieved particle shapes. All of them included inhomogeneities, some of them in a scale of only a few percentages of the volume. In their comparisons to corresponding effectivemedium cases, they found that the overall differences were small and that the hematite content, even if the volume fraction remained below one percent, was the main source for the discrepancies. Interestingly, its effect on single-scattering albedo could not be interpreted to be systematic among these samples. The size dependence of scattering revealed that the size parameter region $x=3 \ldots 8$ was the most sensitive to realistically distributed inhomogeneity and that the impact occurred systematically at moderate to large scattering angles. It is plausible that these findings depend on the scale of inhomogeneity, although this was not tested.

The other inhomogeneity-studies reviewed here are mostly sensitivity studies, where simulations have been used as a laboratory for mapping the 
possible effect of various kinds of inhomogeneity. A systematic study on mixtures of ice and silicate in wavelength-scale particles was performed by [147], who found the motivation to this originally from Solar-System particles, such as cometary dust, but this combination of materials is relevant also in the case of atmospheric particles. They presented a convenient tool, the concave-hull transformation, for generating a concave surface around an arbitrary particle, and utilized it for forming a varying layer of inhomogeneity. As input geometries to the concave hull, they used Gaussian random spheres and aggregates of spheres - note that with the concave hull, both of these result in compact geometries, as presented in Fig. 5, the main difference being that the shapes originating from aggregates have a much higher fraction of the coating material due to the relatively larger volume fraction of the coating. Inhomogeneity was first studied by adding an icy coating on the silicate Gaussian random spheres and aggregates of spheres. This generally decreased the degree of linear polarization and, in the case of aggregates, caused the maximum polarization to shift towards larger scattering angles. Depolarization was not systematically affected as it decreased in the case of Gaussian random spheres but increased for aggregates, particularly for large scattering angles. A comparison to a coated sphere (see [147], Fig. 5) strikingly reminds of the strong resonances of the spherical symmetry typical to a single size parameter; nevertheless, one should keep in mind that when integrated over a size distribution, these resonances tend to average out.

\section{Concluding remarks}

Light scattering by small particles is a cross-disciplinary field with high relevance in many different areas of research. Owing to the large variety of applications, numerous particle models have been developed for modelling light scattering by morphologically complex particles. In this review article our goal was to help the reader to see the forest for the trees by discussing different approaches in relation to the intended purpose of the models.

We can roughly divide particle models into those with (i) highly simplified geometries that do not bear much morphological resemblance to real-world particles, but mainly attempt to mimic the ensemble-averaged optical properties; (ii) geometries that selectively mimic (with various degrees of sophistication) specific morphological features, such as small-scale surface roughness or inhomogeneity; and (iii) highly realistic geometries that are close to being replicas of real-world particles ("numerical laboratories"). Applications 
of light-scattering computations range from aerosol optics modules in large modelling systems (e.g. climate models or remote sensing retrieval schemes) to more fundamental studies and numerical experiments with the goal of deepening our understanding of the impact of morphology on the interaction of light with small particles. Rather than providing an encyclopaedia of published work, we focused on discussing recent trends and key references that helped to illustrate the merits and drawbacks of different model geometries in relation to the designated use of the models.

Spurred by the development of faster computers and improved numerical methods, much progress has been made in recent years in our understanding of complex morphological features, such as surface roughness and aggregation. One of the current frontiers is particle inhomogeneity, which can be important for understanding the optical properties of porous volcanic ash particles, dust particles composed of different minerals, coated LAC aggregates, cosmic dust particles in the form of ice-coated silicate aggregates, or other chemically heterogeneous particles. Future progress will depend on the continued development of numerical methods as well as refined knowledge of the morphology, composition, and dielectric properties of such particles.

[1] M. I. Mishchenko, V. K. Rosenbush, N. N. Kiselev, D. F. Lupishko, V. P. Tishkovets, V. G. Kaydash, I. N. Belskaya, Y. S. Efimov, N. M. Shakhavskoy, Polarimetric Remote Sensing of Solar System Objects, Akademperiodyka, Kyiv, 2010.

[2] D. C. B. Whittet, Dust in the galactic environment, Institute of Physics Publishing, Bristol, 2003.

[3] N. G. Khlebtsov, L. A. Dykman, Optical properties and biomedical applications of plasmonic nanoparticles, J. Quant. Spectrosc. Radiat. Transfer 111 (2010) 1-35.

[4] V. Myroshnychenko, J. Rodríguez-Fernández, I. Pastoriza-Santos, A. M. Funston, C. Novo, P. Mulvaney, L. M. Liz-Marzán, F. J. G. de Abajo, Modelling the optical response of gold nanoparticles, Chem. Soc. Rev. 37 (2008) 1792-1805.

[5] V. Veselago, L. Braginsky, V. Shklover, C. Hafner, Negative refractive index materials, J. Comput. Theoret. Nanosci. 2006 (2006) 1-30. 
[6] A. R. Jones, Light scattering in combustion, in: A. Kokhanovsky (Ed.), Light Scattering Reviews, Springer, Berlin, 2006, pp. 393-444.

[7] S. Merikallio, T. Nousiainen, M. Kahnert, A.-M. Harri, Light scattering by the martian dust analog, palagonite, modeled with ellipsoids, Opt. Express 21 (2013) 17972-17985.

[8] O. Dubovik, A. Sinyuk, T. Lapyonok, B. N. Holben, M. I. Mishchenko, P. Yang, T. F. Eck, H. Volten, O. Muñoz, B. Veihelmann, W. J. van der Zande, J.-F. Leon, M. Sorokin, I. Slutsker, Application of spheroid models to account for aerosol particle nonsphericity in remote sensing of desert dust, J. Geophys. Res. 111 (2006) D11208.

[9] O. Dubovik, T. Lapyonok, Y. J. Kaufman, M. Chin, P. Ginoux, R. A. Kahn, A. Sinyuk, Retrieving global aerosol sources from satellites using inverse modelling, Atmos. Chem. Phys. 8 (2008) 209-250.

[10] M. Z. Jacobson, Strong radiative heating due to the mixing state of black carbon in atmospheric aerosols, Nature 409 (2001) 695-697.

[11] M. I. Mishchenko, , L. D. Travis, A. A. Lacis, Scattering, absorption, and emission of light by small particles, Cambridge University Press, Cambridge, 2002.

[12] M. I. Mishchenko, Electromagnetic scattering by nonspherical particles: a tutorial review, J. Quant. Spectrosc. Radiat. Transfer 110 (2009) 808832 .

[13] T. Rother, M. Kahnert, Electromagnetic wave scattering on nonspherical particles: Basic Methodology and Simulations, 2nd Edition, Springer, Berlin, 2014, http://dx.doi.org/10.1007/978-3-642-36745-8.

[14] M. I. Mishchenko, J. W. Hovenier, L. D. Travis, Light Scattering by Nonspherical Particles, Academic Press, San Diego, 2000.

[15] F. M. Kahnert, Numerical methods in electromagnetic scattering theory, J. Quant. Spectrosc. Radiat. Transfer 79-80 (2003) 775-824.

[16] M. Kahnert, Electromagnetic scattering by nonspherical particles: Recent advances, J. Quant. Spectrosc. Radiat. Transfer 111 (2010) 17881790. 
[17] T. Wriedt, Light scattering theories and computer codes, J. Quant. Spectrosc. Radiat. Transfer 110 (2009) 833-843.

[18] J. M. J. Aan de Brugh, M. Schaap, E. Vignati, F. Dentener, M. Kahnert, M. Sofiev, V. Huijnen, M. C. Krol, The European aerosol budget in 2006, Atmos. Chem. Phys. 11 (2011) 1117-1139.

[19] M. Kahnert, On the observability of chemical and physical aerosol properties by optical observations: Inverse modelling with variational data assimilation, Tellus 61B (2009) 747-755.

[20] J. Tyynelä, E. Zubko, G. Videen, K. Muinonen, Interrelating angular scattering characteristics to internal electric fields for wavelength-scale spherical particles, J. Quant. Spectrosc. Radiat. Transfer 106 (2007) $520-534$.

[21] J. Tyynelä, K. Muinonen, E. Zubko, G. Videen, Interrelating angular scattering characteristics to internal electric fields for gaussian-random sphere particles, J. Quant. Spectrosc. Radiat. Transfer 109 (2008) 22072218.

[22] J. Tyynelä, E. Zubko, K. Muinonen, G. Videen, Interpretation of singleparticle negative polarization at intermediate scattering angles, Appl. Opt. 49 (2010) 5284-5296.

[23] K. Muinonen, J. Tyynelä, E. Zubko, H. Lindqvist, A. Penttilä, G. Videen, Polarization of light backscattered by small particles, J. Quant. Spectrosc. Radiat. Transfer 112 (2011) 2193-2212.

[24] M. I. Mishchenko, Light scattering by size-shape distributions of randomly oriented axially symmetric particles of a size comparable to a wavelength, Appl. Opt. 32 (1993) 4652-4665.

[25] N. V. Voshchinnikov, V. G. Farafonov, Optical properties of spheroidal particles, Astrophys. Space Sci. 204 (1993) 19-86.

[26] M. I. Mishchenko, L. D. Travis, Light scattering by polydispersions of randomly oriented spheroids with sizes comparable to wavelengths of observation, Appl. Opt. 33 (1994) 7206-7225. 
[27] M. I. Mishchenko, L. D. Travis, R. A. Kahn, R. A. West, Modeling phase functions for dustlike tropospheric aerosols using a shape mixture of randomly oriented polydisperse spheroids, J. Geophys. Res. 102 (1997) 16,831-16,847.

[28] F. M. Schulz, K. Stamnes, J. J. Stamnes, Modeling the radiative transfer properties of media containing particles of moderately and highly elongated shape, Geophys. Res. Lett. 25 (1998) 4481-4484.

[29] F. M. Schulz, K. Stamnes, J. J. Stamnes, Shape-dependence of the optical properties in size-shape distributions of randomly oriented prolate spheroids, including highly elongated shapes, J. Geophys. Res. 104 (1999) 9413-9421.

[30] N. T. Zakharova, M. I. Mishchenko, Scattering properties of needle-like and plate-like ice spheroids with moderate size parameters, Appl. Opt. 39 (2000) 5052-5057.

[31] N. V. Voshchinnikov, V. B. Il'in, T. Henning, , B. Michel, V. G. Farafonov, Extinction and polarization of radiation by absorbing spheroids: shape/size effects and benchmark results, J. Quant. Spectrosc. Radiat. Transfer 65 (2000) 877-893.

[32] M. I. Mishchenko, L. D. Travis, A. Macke, Scattering of light by polydisperse, randomly oriented, finite circular cylinders, Appl. Opt. 35 (1996) 4927-4940.

[33] N. T. Zakharova, M. I. Mishchenko, Scattering by randomly oriented thin ice disks with moderate equivalent-sphere size parameters, J. Quant. Spectrosc. Radiat. Transfer 70 (2001) 465-471.

[34] A. Mugnai, W. J. Wiscombe, Scattering from nonspherical Chebyshev particles. 1: cross sections, single scattering albedo, asymmetry factor, and backscattering fraction, Appl. Opt. 25 (1986) 1235-1244.

[35] M. I. Mishchenko, L. D. Travis, Light scattering by polydisperse, rotationally symmetric nonspherical particles: linear polarization, J. Quant. Spectrosc. Radiat. Transfer 51 (1994) 759-778. 
[36] J. Ding, L. Xu, Convergence of the T-matrix approach for randomly oriented, nonabsorbing, nonspherical Chebyshev particles, J. Quant. Spectrosc. Radiat. Transfer 63 (1999) 163-174.

[37] T. Rother, K. Schmidt, J. Wauer, V. Shcherbakov, J.-F. Gaeyt, Light scattering on Chebyshev particles of higher order, Appl. Opt. 45 (2006) 6030-6037.

[38] D. Petrov, Y. Shkuvatov, G. Videen, Analytical light-scattering solution for Chebyshev particles, J. Opt. Soc. Am. A 24 (2007) 1103-1119.

[39] J.-C. Auger, G. Fernandes, K. Aptowicz, Y.-L. Pan, R. Chang, Influence of surface roughness on the elastic-light scattering patterns of micron-sized aerosol particles, Appl. Phys. B 99 (2010) 229-234.

[40] A. S. Eddington, The Internal Constitution of the Stars, Cambridge University Press, London, 1926.

[41] M. Kahnert, T. Nousiainen, H. Lindqvist, Models for integrated and differential scattering optical properties of encapsulated light absorbing carbon aggregates, Opt. Express 21 (2013) 7974-7992.

[42] M. I. Mishchenko, V. P. Tishkovets, L. D. Travis, B. Cairns, J. M. Dlugach, L. Liu, V. K. Rosenbush, N. N. Kiselev, Electromagnetic scattering by a morphologically complex object: Fundamental concepts and common misconceptions, J. Quant. Spectrosc. Radiat. Transfer 112 (2011) 671-692.

[43] W. J. Wiscombe, A. Mugnai, Scattering from nonspherical Chebyshev particles. 2: Means of angular scattering patterns, Appl. Opt. 27 (1988) $2405-2421$.

[44] A. Mugnai, W. J. Wiscombe, Scattering from nonspherical Chebyshev particles. 3: Variability in angular scattering patters, Appl. Opt. 28 (1989) 3061-3073.

[45] T. Nousiainen, K. Muinonen, Surface-roughness effects on singlescattering properties of wavelength-scale particles, J. Quant. Spectrosc. Radiat. Transfer 106 (2007) 389-397. doi:10.1016/j.jqsrt.2007.01.024. 
[46] M. Kahnert, T. Nousiainen, P. Mauno, On the impact of non-sphericity and small-scale surface roughness on the optical properties of hematite aerosols, J. Quant. Spectrosc. Radiat. Transfer 112 (2011) 1815-1824.

[47] M. Kahnert, T. Rother, Modeling optical properties of particles with small-scale surface roughness: combination of group theory with a perturbation approach, Opt. Express 19 (2011) 11138-11151.

[48] M. Kahnert, T. Nousiainen, M. A. Thomas, J. Tyynelä, Light scattering by particles with small-scale surface roughness: comparison of four classes of model geometries, J. Quant. Spectrosc. Radiat. Transfer 113 (2012) 2356-2367.

[49] K. Muinonen, T. Nousiainen, P. Fast, K. Lumme, J. I. Peltoniemi, Light scattering by gaussian random particles: ray optics approximation, J. Quant. Spectrosc. Radiat. Transfer 55 (1996) 577-601.

[50] M. Kahnert, The t-matrix code tsym for homogeneous dielectric particles with finite symmetries, J. Quant. Spectrosc. Radiat. Transfer 123 (2013) 62-78.

[51] E. Zubko, K. Muinonen, Y. Shkuratov, G. Videen, T. Nousiainen, Scattering of light by roughened Gaussian random particles, J. Quant. Spectrosc. Radiat. Transfer 106 (2007) 604-615.

[52] P. C. Waterman, Matrix formulation of electromagnetic scattering, Proc. IEEE 53 (1965) 805-812.

[53] M. Kahnert, Irreducible representations of finite groups in the T matrix formulation of the electromagnetic scattering problem, J. Opt. Soc. Am. A 22 (2005) 1187-1199.

[54] M. Kahnert, T-matrix computations for particles with high-order finite symmetries, J. Quant. Spectrosc. Radiat. Transfer 123 (2013) 79-91.

[55] M. A. Yurkin, M. Kahnert, Light scattering by a cube: accuracy limits of the discrete dipole approximation and the t-matrix method, J. Quant. Spectrosc. Radiat. Transfer 123 (2013) 176-183.

[56] M. Mishchenko, J. Dlugach, D. Mackowski, Light scattering by wavelength-sized particles dusted with subwavelength-sized grains, Optics Letters 36 (2011) 337-339. 
[57] J. M. Dlugach, M. I. Mishchenko, D. W. Mackowski, Scattering and absorption properties of polydisperse wavelegth-sized particles covered with much smaller grains, J. Quant. Spectrosc. Radiat. Transfer 113 (2012) 2351-2355.

[58] D. W. Mackowski, M. I. Mishchenko, Calculation of the T matrix and the scattering matrix for ensembles of spheres, J. Opt. Soc. Am. A 13 (1996) 2266-2278.

[59] T. Nousiainen, Impact of particle shape on refractive-index dependence of scattering in resonance domain, J. Quant. Spectrosc. Radiat. Transfer 108 (2007) 464-473. doi:10.1016/j.jqsrt.2007.07.008.

[60] T. Nousiainen, M. Kahnert, H. Lindqvist, Can particle shape information be retrieved from light-scattering observations using spheroidal model particles?, J. Quant. Spectrosc. Radiat. Transfer 112 (2011) 2213-2225. doi:10.1016/j.jqsrt.2011.05.008.

[61] F. M. Kahnert, Reproducing the optical properties of fine desert dust aerosols using ensembles of simple model particles, J. Quant. Spectrosc. Radiat. Transfer 85 (2004) 231-249.

[62] H. Lindqvist, O. Jokinen, K. Kandler, D. Scheuvens, T. Nousiainen, Single scattering by realistic, inhomogeneous mineral dust particles with stereogrammetric shapes, Atmos. Chem. Phys. 14 (2014) 143-157. doi:10.5194/acp-14-143-2014.

[63] S. Merikallio, H. Lindqvist, T. Nousiainen, M. Kahnert, Modelling light scattering by mineral dust using spheroids: assessment of applicability, Atmos. Chem. Phys. 11 (2011) 5347-5363.

[64] K. Schmidt, J. Wauer, T. Rother, T. Trautmann, Scattering database for spheroidal particles, Appl. Opt. 48 (2009) 2154-2164.

[65] Z. Meng, P. Yang, G. W. Kattawar, L. Bi, K. N. Liou, , I. Laszlo, Single-scattering properties of tri-axial ellipsoidal mineral dust aerosols: A database for application to radiative transfer calculations, J. Aerosol Sci 41 (2010) 501-512. 
[66] O. Dubovik, B. N. Holben, T. Lapyonok, A. Sinyuk, M. I. Mishchenko, P. Yang, I. Slutsker, Non-spherical aerosol retrieval method employing light scattering by spheroids, Geophys. Res. Lett. 29 (2002) 54-1-54-4.

[67] R. C. Levy, L. A. Remer, O. Dubovik, Global aerosol optical properties and application to Moderate Resolution Imaging Spectroradiometer aerosol retrieval over land, J. Geophys. Res. 112 (2007) 3210. doi:doi:10.1029/2006JD007815.

[68] Y. M. Govaerts, S. Wagner, A. Lattanzio, P. Watts, Joint retrieval of surface reflectance and aerosol optical depth from MSG/SEVIRI observations with an optimal estimation approach: 1. Theory, J. Geophys. Res. 115 (2010) D02203. doi:10.1029/2009JD011779.

[69] S. C. Wagner, Y. M. Govaerts, A. Lattanzio, Joint retrieval of surface reflectance and aerosol optical depth from MSG/SEVIRI observations with an optimal estimation approach: 2. Implementation and evaluation, J. Geophys. Res. 115 (2010) D02204. doi:10.1029/2009JD011780.

[70] O. Dubovik, M. Herman, A. Holdak, T. Lapyonok, D. Tanré, J. L. Deuzé, F. Ducos, A. Sinyuk, A. Lopatin, Statistically optimized inversion algorithm for enhanced retrieval of aerosol properties from spectral multiangle polarimetric satellite observations, Atmos. Meas. Tech. 4 (2011) 9751018.

[71] H. Volten, O. Muñoz, E. Rol, J. F. de Haan, W. Vassen, J. W. Hovenier, K. Muinonen, T. Nousiainen, Scattering matrices of mineral aerosol particles at $441.6 \mathrm{~nm}$ and $632.8 \mathrm{~nm}$, J. Geophys. Res. 106 (2001) 1737517401.

[72] C. Cattrall, J. Reagan, K. Thome, O. Dubovik, Variability of aerosol and spectral lidar and backscatter and extinction ratios of key aerosol types derived from selected Aerosol Robotic Network locations, J. Geophys. Res. 110 (2005) D10SA11. doi:10.1029/2004JD005124.

[73] I. Veselovskii, O. Dubovik, A. Kolgotin, T. Lapyonok, P. D. Girolamo, D. Summa, D. N. Whiteman, M. Mishchenko, D. Tanre, Application of randomly oriented spheroids for retrieval of dust particle parameters from multiwavelength lidar measurements, J. Geophys. Res. 115 (2010) D21203. doi:10.1029/2010JD014139. 
[74] P. Di Girolamo, D. Summa, R. Bhawar, T. D. Iorio, M. Cacciani, I. Veselovskii, O. Dubovik, A. Kolgotin, Raman lidar observations of a saharan dust outbreak event: Characterization of the dust optical properties and determination of particle size and microphysical parameters, Atmos. Env. 50 (2012) 66-78.

[75] G. L. Schuster, M. Vaughan, D. MacDonnell, W. Su, D. Winker, O. Dubovik, T. Lapyonok, C. Trepte, Comparison of CALIPSO aerosol optical depth retrievals to AERONET measurements, and a climatology for the lidar ratio of dust, Atmos. Chem. Phys 12 (2012) 74317452.

[76] J. Wagner, A. Ansmann, U. Wandinger, P. Seifert, A. Schwarz, M. Tesche, A. Chaikovsky, , O. Dubovik, Evaluation of the Lidar/Radiometer Inversion Code (LIRIC) to determine microphysical properties of volcanic and desert dust, Atmos. Meas. Tech. 6 (2013) $1707-1724$.

[77] A. Lopatin, O. Dubovik, A. Chaikovsky, P. Goloub, T. Lapyonok, D. Tanr, , P. Litvinov, Enhancement of aerosol characterization using synergy of lidar and sun-photometer coincident observations: the GARRLiC algorithm, Atmos. Meas. Tech. 6 (2013) 2065-2088.

[78] L. Bi, P. Yang, G. Kattawar, R. Kahn, Single-scattering properties of triaxial ellipsoidal particles for a size parameter range from the Rayleigh to geometric-optics regimes, Appl. Opt. 48 (2009) 114-126.

[79] T. Nousiainen, M. Kahnert, B. Veihelmann, Light scattering modeling of small feldspar aerosol particles using polyhedral prisms and spheroids, J. Quant. Spectrosc. Radiat. Transfer 101 (2006) 471-487.

[80] L. Bi, P. Yang, G. Kattawar, R. Kahn, Modeling optical properties of mineral aerosol particles by using nonsymmetric hexahedra, Appl. Opt. 49 (2010) 334-342.

[81] K. Muinonen, E. Zubko, J. Tyynelä, Y. Shkuratov, G. Videen, Light scattering by Gaussian random particles with discrete-dipole approximation, J. Quant. Spectrosc. Radiat. Transfer 106 (2007) 360-377. doi:10.1016/j.jqsrt.2007.01.049. 
[82] T. Nousiainen, K. Muinonen, J. Avelin, A. Sihvola, Microwave backscattering by nonspherical ice particles at $5.6 \mathrm{GHz}$ using secondorder perturbation series, J. Quant. Spectrosc. Radiat. Transfer 70 (2001) 639-661.

[83] T. Nousiainen, E. Zubko, H. Lindqvist, M. Kahnert, J. Tyynelä, Comparison of scattering by different nonspherical, wavelength-scale particles, J. Quant. Spectrosc. Radiat. Transfer 2012 (2012) 2391-2405.

[84] C. Liu, R. L. Panetta, P. Yang, A. Macke, A. J. Baran, Modeling the scattering properties of mineral aerosols using concave fractal polyhedra, Appl. Opt. 52 (2012) 640-652.

[85] W. Tape, Atmospheric Halos, Americal Geophysical Union, 1994.

[86] D. L. Mitchell, K. Chai, Y. Liu, A. J. Heymsfield, Y. Dong, Modeling cirrus clouds. Part I: treatment of bimodal size spectra and case study analysis, J. Atmos. Sci. 53 (1996) 2952-2966.

[87] P. R. Buseck, M. Pósfai, Airborne minerals and related aerosol particles: Effects on climate and the environment, Proc. Natl. Acad. Sci. USA 96 (1999) 3372-3379.

[88] P. Yang, K. N. Liou, W. P. Arnott, Extinction efficiency and singlescattering albedo for laboratory and natural cirrus clouds, J. Geophys. Res. 102 (1997) 21,825-21,835.

[89] A. Macke, M. I. Mishchenko, B. Cairns, The influence of inclusions on light scattering by large ice particles, J. Geophys. Res. 101 (1996) $23,311-23,316$.

[90] A. Macke, P. N. Francis, G. M. McFarquhar, S. Kinne, The role of ice particle shapes and size distributions in single scattering properties of cirrus clouds, J. Atmos. Sci. 55 (1998) 2874-2883.

[91] A. Macke, J. Mueller, E. Raschke, Single scattering properties of atmospheric ice crystals, J. Atmos. Sci. 53 (1996) 2813-2825.

[92] P. Yang, K. N. Liou, Light scattering by ice crystals of complex shapes, in: Ninth Conference on Atmospheric Radiation, Long Beach, California, February 2-7, 1997, Am. Meteorol. Soc., Boston, Mass., 1997, pp. $373-377$. 
[93] F. M. Kahnert, J. J. Stamnes, K. Stamnes, Using simple particle shapes to model the Stokes scattering matrix of ensembles of wavelength-sized particles with complex shapes: possibilities and limitations, J. Quant. Spectrosc. Radiat. Transfer 74 (2002) 167-182.

[94] P. Yang, K. N. Liou, M. I. Mishchenko, B.-C. Gao, Efficient finitedifference time-domain scheme for light scattering by dielectric particles: application to aerosols, Appl. Opt. 39 (2000) 3727-3737.

[95] M. Matricardi, The inclusion of aerosols and clouds in RTIASI, the ECMWF fast radiative transfer model for the infrared atmospheric sounding interferometer, Tech. Rep. 474, ECMWF Research Dept. Tech. Memo., Reading, UK (2005).

[96] G. David, B. Thomas, T. Nousiainen, A. Miffre, P. Rairoux, Retrieving simulated volcanic, desert dust and sea-salt particle properties from two/three-component particle mixtures using UV-VIS polarization lidar and T matrix, Atmos. Chem. Phys. 13 (2013) 6757-6776. doi:10.5194/acp-13-6757-2013.

[97] T. C. Bond, R. W. Bergstrom, Light absorption by carbonaceous particles: An investigative review, Aerosol Sci. Technol. 40 (2006) 27-67.

[98] O. Muñoz, H. Volten, J. F. de Haan, W. Vassen, J. W. Hovenier, Experimental determination of scattering matrices of randomly oriented fly ash and clay particles at 442 and 633 nm, J. Geophys. Res. 106 (2001) 22833-22844.

[99] A. J. Baran, L. C. Labonnote, On the reflection and polarisation properties of ice cloud, J. Quant. Spectrosc. Radiat. Transfer 100 (2006) $41-54$.

[100] Y. Okada, I. Mann, T. Mukai, M. Köhler, Extended calculation of polarization and intensity of fractal aggregates based on rigorous method for light scattering simulations with numerical orientation averaging, J. Quant. Spectrosc. Radiat. Transfer 109 (2008) 2613-2627.

[101] E. M. Purcell, C. R. Pennypacker, Scattering and absorption of light by nonspherical dielectric grains, Astrophys. J. 186 (1973) 705-714. 
[102] C. F. Bohren, D. R. Huffman, Absorption and Scattering of Light by Small Particles, Wiley-VCH, Weinheim, 1983.

[103] Y. Zhao, L. Ma, Assessment of two fractal scattering models for the prediction of the optical characteristics of soot aggregates, J. Quant. Spectrosc. Radiat. Transfer 110 (2009) 315-322.

[104] D. W. Mackowski, Calculation of total cross sections of multiple-sphere clusters, J. Opt. Soc. Am. A 11 (1994) 2851-2861.

[105] Y. Xu, B. A. S. Gustafson, A generalized multiparticle Mie solution: further experimental verification, J. Quant. Spectrosc. Radiat. Transfer 70 (2001) 395-419.

[106] D. W. Mackowski, M. I. Mishchenko, A multiple sphere T-matrix Fortran code for use on parallel computer clusters, J. Quant. Spectrosc. Radiat. Transfer 112 (2011) 2182-2192.

[107] C. M. Sorensen, Light scattering by fractal aggregates: A review, Aerosol Sci. Technol. 35 (2001) 648-687.

[108] M. Kahnert, On the discrepancy between modelled and measured mass absorption cross sections of light absorbing carbon aerosols, Aerosol Sci. Technol. 44 (2010) 453-460.

[109] L. Liu, M. I. Mishchenko, Effects of aggregation on scattering and radiative properties of soot aerosols, J. Geophys. Res. 110 (2005) D11211.

[110] L. Liu, M. I. Mishchenko, Scattering and radiative properties of complex soot and soot-containing aggregate particles, J. Quant. Spectrosc. Radiat. Transfer 106 (2007) 262-273.

[111] L. Liu, M. I. Mishchenko, W. P. Arnott, A study of radiative properties of fractal soot aggregates using the superposition $T$-matrix method, J. Quant. Spectrosc. Radiat. Transfer 109 (2008) 2656-2663.

[112] M. Kahnert, Modelling the optical and radiative properties of freshly emitted light absorbing carbon within an atmospheric chemical transport model, Atmos. Chem. Phys. 10 (2010) 1403-1416. 
[113] M. Kahnert, Numerically exact computation of the optical properties of light absorbing carbon aggregates for wavelength of $200 \mathrm{~nm} 12.2$ $\mu \mathrm{m}$, Atmos. Chem. Phys. 10 (2010) 8319-8329.

[114] M. Kahnert, A. Devasthale, Black carbon fractal morphology and short-wave radiative impact: a modelling study, Atmos. Chem. Phys. 11 (2011) 11745-11759.

[115] Y. Takano, K. N. Liou, M. Kahnert, P. Yang, The single-scattering properties of black carbon aggregates determined from the geometricoptics surface-wave approach and the t-matrix method, J. Quant. Spectrosc. Radiat. Transfer 125 (2013) 51-56.

[116] R. Jullien, R. Botet, Aggregation and Fractal Aggregates, World Scientific, Singapore, 1987.

[117] K. A. Fuller, W. C. Malm, S. M. Kreidenweis, Effects of mixing on extinction by carbonaceous particles, J. Geophys. Res. 104 (1999) 1594115954.

[118] L. H. van Poppel, H. Friedrich, J. Spinsby, S. H. Chung, J. H. Seinfeld, P. R. Buseck, Electron tomography of nanoparticle clusters: Implications for atmospheric lifetimes and radiative forcing of soot, Geophys. Res. Lett. 32 (2005) L24811, doi:10.1029/2005GL024461.

[119] K. Adachi, S. H. Chung, H. Friedrich, P. R. Buseck, Fractal parameters of individual soot particles determined using electron tomography: Implications for optical properties, J. Geophys. Res. 112 (2007) D14202, doi:10.1029/2006JD008296.

[120] K. Adachi, S. Chung, P. R. Buseck, Shapes of soot aerosol particles and implications for their effects on climate, J. Geophys. Res. 115 (2010) D15206, doi:10.1029/2009JD012868.

[121] T. Wriedt, J. Wilkens, J. Hellmers, Differentiating between sintered and non-sintered aggregates, in: K. Muinonen, A. Penttilä, H. Lindqvist, T. Nousiainen, G. Videen (Eds.), Electromagnetic and Light Stattering XII, University of Helsinki, Helsinki, 2010, pp. 322325 . 
[122] K. Skorupski, J. Mroczka, N. Riefler, H. Oltmann, S. Will, T. Wriedt, Impact of morphological parameters onto simulated light scattering patterns, J. Quant. Spectrosc. Radiat. Transfer 119 (2013) 53-66.

[123] T. C. Bond, G. Habib, R. W. Bergstrom, Limitations in the enhancement of visible light absorption due to mixing state, J. Geophys. Res. 111 (2006) D20211, doi:10.1029/2006JD007315.

[124] C. D. Cappa, T. B. Onasch, P. Massoli, D. R. Worsnop, T. S. Bates, E. S. Cross, P. Davidovits, J. Hakala, K. L. Hayden, B. T. Jobson, k. R. Kolesar, D. A. Lack, B. M. Lerner, S.-M. Li, D. Mellon, I. Nuaaman, J. S. O. T. Petäjä, P. K. Quinn, C. Song, R. Subramanian, E. J. Williams, R. A. Zaveri, Radiative absorption enhancements due to the mixing state of atmospheric black carbon, Science 337 (2012) 1078-1081.

[125] A. Even, A. Smekens, A. Khlystov, P. Berghmans, R. van Grieken, H. M. ten Brink, Morphology of internally mixed aerosol of ammonium sulfate and soot, J. Aerosol Sci. 29 (1998) 753-754.

[126] M. Schnaiter, C. Linke, O. Moehler, K.-H. Naumann, H. Saathoff, R. Wagner, U. Schurath, , B. Wehner, Absorption amplification of black carbon internally mixed with secondary organic aerosol, J. Geophys. Res. 110 (2005) D19204, doi:10.1029/2005JD006046.

[127] B. Scarnato, S. Vahidinia, D. T. Richard, T. W. Kirchstetter, Effects of internal mixing and aggregate morphology on optical properties of black carbon using a discrete dipole approximation model, Atmos. Chem. Phys. 13 (2013) 5089-5101.

[128] E. F. Mikhailov, S. S. Vlasenko, I. A. Podgorny, V. Ramanathan, C. E. Corrigan, Optical properties of soot-water drop agglomerates: An experimental study, J. Geophys. Res. 111 (2006) D07209, doi:10.1029/2005JD006389.

[129] H. Chang, T. T. Charalampopoulos, Determination of the wavelength dependence of refractive indices of flame soot, Proc. R. Soc. Lond. A 430 (1990) 577-591.

[130] M. Hess, P. Koepke, I. Schult, Optical properties of aerosols and clouds: The software package OPAC, Bull. Am. Met. Soc. 79 (1998) 831-844. 
[131] M. Kahnert, Variational data analysis of aerosol species in a regional CTM: background error covariance constraint and aerosol optical observation operators, Tellus 60B (2008) 753-770.

[132] G. Videen, P. Chýlek, Scattering by a composite sphere with an absorbing inclusion and effective medium approximations, Opt. Commun. 158 (1998) 1-6.

[133] P. Chýlek, G. Videen, D. J. W. Geldart, J. S. Dobbie, H. C. W. Tso, Effective medium approximations for heterogeneous particles, in: M. I. Mishchenko, J. W. Hovenier, L. D. Travis (Eds.), Light scattering by nonspherical particles, Academic Press, San Diego, 2000, pp. 274-308.

[134] T. P. Ackerman, O. B. Toon, Absorption of visible radiation in atmospheres containing mixtures of absorbing and nonabsorbing particles, Appl. Opt. 20 (1981) 3661-3668.

[135] K. A. Fuller, Scattering and absorption cross sections of compounded spheres III. spheres containing arbitrarily located spherical inhomogeneities, J. Opt. Soc. Am. A 12 (1995) 893-904.

[136] M. I. Mishchenko, L. Liu, L. D. Travis, A. A. Lacis, Scattering and radiative properties of semi-external versus external mixtures of different aerosol types, J. Quant. Spectrosc. Radiat. Transfer 88 (2004) 139-147.

[137] M. Kocifaj, G. Videen, Optical behavior of composite carbonaceous aerosols: DDA and EMT approaches, J. Quant. Spectrosc. Radiat. Transfer 109 (2008) 1404-1416.

[138] A. Worringen, M. Ebert, T. Trautmann, S. Weinbruch, G. Helas, Optical properties of internally mixed ammonium sulfate and soot particlesa study of individual aerosol particles and ambient aerosol populations, Appl. Opt. 47 (2008) 3835-3845.

[139] K. Adachi, P. R. Buseck, Internally mixed soot, sulfates, and organic matter in aerosol particles from Mexico City, Atmos. Chem. Phys. 8 (2008) 6469-6481.

[140] M. Kahnert, T. Nousiainen, H. Lindqvist, M. Ebert, Optical properties of light absorbing carbon aggregates mixed with sulfate: assessment of 
different model geometries for climate forcing calculations, Opt. Express 20 (2012) 10042-10058.

[141] M. Z. Jacobson, A physically-based treatment of elemental carbon optics: Implications for global direct forcing of aerosols, Geophys. Res. Lett 27 (2000) 217-220.

[142] M. A. Iatì, R. Saija, F. Borghese, P. Denti, C. Cecchi-Pestellini, D. A. Williams, Stratified dust grains in the interstellar medium i. an accurate computational method for calculating their optical properties, Mon. Not. R. Astron. Soc. 384 (2008) 591-598.

[143] H. Lindqvist, T. Nousiainen, E. Zubko, O. Muñoz, Optical modeling of vesicular volcanic ash particles, J. Quant. Spectrosc. Radiat. Transfer 112 (2011) 1871-1880.

[144] A. Kylling, M. Kahnert, H. Lindqvist, T. Nousiainen, Volcanic ash infrared signature: Realistic ash particle shapes compared to spherical ash particles, Atmos. Meas. Tech. Discuss. 6 (2013) 8937-8958.

[145] R. Vilaplana, F. Moreno, A. Molina, Study of the sensitivity of size-averaged scattering matrix elements of nonspherical particles to changes in shape, porosity and refractive index, J. Quant. Spectrosc. Radiat. Transfer 100 (2006) 415-428. doi:10.1016/j.jqsrt.2005.11.068.

[146] K. Muinonen, T. Nousiainen, H. Lindqvist, O. Muñoz, G. Videen, Light scattering by Gaussian particles with internal inclusions and roughened surfaces using ray optics, J. Quant. Spectrosc. Radiat. Transfer 110 (2009) 1628-1639. doi:10.1016/j.jqsrt.2009.03.012.

[147] H. Lindqvist, K. Muinonen, T. Nousiainen, Light scattering by coated Gaussian and aggregate particles, J. Quant. Spectrosc. Radiat. Transfer 110 (2009) 1398-1410. doi:10.1016/j.jqsrt.2009.01.015.

\section{Acknowledgements}

M. Kahnert acknowledges funding from the Swedish Research Council (project 621-2011-3346). T. Nousiainen and H. Lindqvist acknowledge funding by the Academy of Finland and the Finnish Funding Agency for Technology and Innovation (Tekes). We are grateful to Oleg Dubovik, Mike Wolff, 
and another anonymous reviewer for providing very helpful comments to this article. 